\title{
SPARC Interacts with Actin in Skeletal Muscle in Vitro and in Vivo
}

Louise H. Jørgensen, ${ }^{\dagger \dagger}$ Pia L. Jepsen, ${ }^{*}$ Anders Boysen, ${ }^{\ddagger}$ Line B. Dalgaard, ${ }^{\star \S}$ Lars G. Hvid, ${ }^{\S}$ Niels $\emptyset$ rtenblad,,$\S$ Dea Ravn, * Jeeva Sellathurai, ${ }^{*}$ Jakob Møller-Jensen, ${ }^{\ddagger}$ Hanns Lochmüller, ${ }^{\dagger}$ and Henrik D. Schrøder*

\begin{abstract}
From the Department of Pathology, * Institute of Clinical Research, University of Southern Denmark Muscle Research Cluster, the Department of Biochemistry and Molecular Biology, ${ }^{\ddagger}$ and the Institute of Sports Science and Clinical Biomechanics, ${ }^{\S}$ University of Southern Denmark Muscle Research Cluster, University of Southern Denmark, Odense, Denmark; the Institute of Genetic Medicine, ${ }^{\dagger}$ Newcastle University, Newcastle upon Tyne, United Kingdom
\end{abstract}

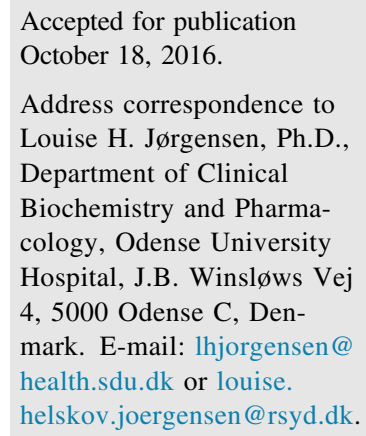

The myopathies constitute a group of degenerative disorders that affect the skeletal muscle system, thereby causing varying degrees of weakness and impairment, depending on the underlying molecular mechanism and severity state. ${ }^{1}$ We previously found that the multifunctional $\mathrm{Ca}^{2+}$-binding protein secreted protein acidic and rich in cysteine (SPARC) is expressed by regenerating muscle fibers and myoblasts in patients with different types of myopathies, such as Duchenne muscular dystrophy, inclusion body myositis, and congenital muscular dystrophy. ${ }^{2}$ Moreover, expression level of SPARC correlated with damage severity of the affected muscles. ${ }^{2}$ In normal adult muscle, SPARC is not detectable except for a very few cells located in the interstitium. ${ }^{2}$ The finding of SPARC within damaged muscle fibers and myoblasts is intriguing because SPARC has generally been described in many tissues as an extracellular located protein involved in regulation of matrix reorganization, cell adhesion, and proliferation. ${ }^{3}$
Substitution of muscle tissue for fat and connective tissue is prominent, especially in severe dystrophies, where continued cycles of degeneration and regeneration exhaust the satellite cell pool. ${ }^{4}$ SPARC has been associated with a number of fibrotic conditions, including scleroderma, ${ }^{5}$ lung, ${ }^{6}$ liver, ${ }^{7}$ and adipose tissue $^{8}$ fibrosis. Hence, SPARC expression in myopathies could relate to formation of fibrosis, ${ }^{9}$ possibly through extracellular matrix reorganization, ${ }^{10}$ because SPARC is known to bind thrombospondin, fibrillar collagens I, III, V, and basement membrane collagen type IV. ${ }^{11,12}$

\footnotetext{
Supported by Villum Kann Rasmussen (L.H.J.), The Danish Council for Independent Research Medical Sciences grant 0602-01704B (L.H.J.), and the Agnes and Poul Friis Foundation (L.H.J.).

Disclosures: None declared.

Current address of L.H.J., Odense University Hospital, Odense, Denmark.
} 
During mouse development SPARC is detected in the somites, ${ }^{13}$ and in human fetal muscle, we detect a prominent expression of SPARC in early myotubes with expression decreasing as the muscles mature. ${ }^{2}$ SPARC null mice have severe cataracts ${ }^{14}$ and osteopenia, ${ }^{15}$ but they do not appear to have an overt muscle phenotype, ${ }^{14}$ even though it was suggested that SPARC null mice have a decreased muscle mass. ${ }^{16}$

The potential effect on muscle mass by lack of SPARC was further implied by a study that found that siRNA knock down of SPARC in vivo causes myofiber atrophy, and it was suggested that reduced SPARC in aging muscle might correlate with sarcopenia. ${ }^{17}$ Further substantiating a role for SPARC in skeletal muscle function is the observation that eradication of SPARC in Xenopus laevis resulted in developmental defects in many tissues after hatching, including the myotome, which appears disordered, ${ }^{18}$ as well as a study that found that in Caenorhabditis elegans the SPARC homologue is expressed by body wall and sex muscle cells, and overexpression results in deformed embryos, affected mobility, and even paralysis. ${ }^{19}$

In cell culture, we detected SPARC during proliferation with a prominent up-regulation during differentiation in both mouse and human myoblasts. ${ }^{2,20}$ In line with the effects of overexpression of SPARC in C. elegans, we have observed that overexpression of SPARC in $\mathrm{C} 2 \mathrm{C} 12$ cells inhibits muscle cell differentiation. ${ }^{20}$ Contrary, another study found that the addition of SPARC protein to muscle cells facilitated differentiation, ${ }^{21}$ suggesting diverse effects, depending on SPARC being present intracellularly or extracellularly and supporting that SPARC could have a direct cellular function.

An actual shuttling effect of SPARC has recently been found, where SPARC appears to bring extracellular matrix proteins into fibroblasts by acting as a chaperone and facilitating extracellular matrix disassembly, ${ }^{22}$ thereby serving a specific role within the cells. The ability of SPARC to function as a chaperone is also implied by SPARC's ability to mediate a cell survival effect, which has been observed in mouse lens epithelial cells ${ }^{23}$ and human cancers, such as melanoma and leukemia. ${ }^{24,25}$ Furthermore, SPARC has also been described as a heat shock protein (HSP), ${ }^{26}$ and its expression has been associated with other stress-induced proteins, such as HSP47 and HSP70. ${ }^{27,28}$ Thus, a general role of SPARC could be of a physiologic nature with the specific function to protect cells or tissues during a temporarily stressful or unfit state. We investigated the specific function of SPARC in skeletal muscle. We found that SPARC interacts directly with actin and that the absence of SPARC results in a changed expression pattern of the different actin isoforms in vitro and force generation deficit after muscle fatigue in vitro. Our results therefore indicate a new mechanistic intracellular role for SPARC, where SPARC might serve to modulate cytoskeletal structure and function in skeletal muscle.

\section{Materials and Methods}

\section{Mice and Animal Experiments}

C57BL/10ScSn- $D M D^{m d x} \mathrm{~J}(m d x)$ mice, wild-type control C57BL/10 (wt), and B6;129S-SPARC ${ }^{\mathrm{tm} 1 \mathrm{Hwe} / \mathrm{J}}$ mice were obtained from The Jackson Laboratory. B6;129S$S P A R C^{t m 1 H w e / J}$ mice were bred as heterozygotes $\left(S P A R C^{+/-}\right)$ to obtain wild-type littermate controls $\left(S P A R C^{+/+}\right)$and SPARC knockout mice $\left(S P A R C^{-1-}\right)$; genotyping was performed according to The Jackson Laboratory protocol (Bar Harbor, ME).

Tibialis anterior (TA) were harvested from 7- to 10-weekold and 24- to 30-week-old $m d x$ and wt mice for histologic examination ( $n=4$ to 5 in each group).

Extensor digitorum longus (EDL) and soleus muscle were excised from $S P A R C^{-/-}$and $S P A R C^{+/+}$adult mice after weighing for in vitro force measurements ( $n=6$ to 11) and myofiber phenotype and diameter measurements ( $n=3$ to 4 ).

Muscle regeneration was studied after an injection with $50 \mu \mathrm{L}$ of cardiotoxin $(10 \mu \mathrm{mol} / \mathrm{L}$; Sigma-Aldrich, St Louis, $\mathrm{MO})$ in the right TA muscle of adult $S P A R C^{+/+}$and $S P A R C^{-1-}$ mice. The left TA muscle served as contralateral control. Mice were sacrificed with cervical dislocation after 3 and 7 days ( $n=5$ in each group) and TAs harvested.

An exercise study (short-term eccentric running) and force grip measurements before and after running were performed in adult $S P A R C^{+/+}$and $S P A R C^{-/-}$mice $(n=3$ in each group). The mice were placed in an electric treadmill (IITC Life Science 800 Series Treadmill; IITC Inc. Life Science, Woodland Hills, CA) adjusted to a $15^{\circ}$ angle to allow downhill running and thus subject the mice to eccentric (lengthening) strain. The top speed was set at $18 \mathrm{~m}$ per minute with an initial speed of $1 \mathrm{~m}$ per minute and a ramp speed of 60 seconds. The duration of the run was 25 minutes. Before the actual short-term running experiments, the mice were familiarized with the treadmill a few days before so they knew what to expect and what to do when placed in the treadmill.

Force grip measurements were collected before the short-term exercise regimen and after running (1 minute, 10 minutes, 30 minutes). The mice were held by the tail and moved over a T-bar mounted on a force measuring devise (IITC Life Science Grip Strength Meter), and the grip force was recorded when the mice were holding the bar with both front paws and pulled before letting go of the bar. At least 5 measurements were performed, and the maximum force recorded was used.

All animal experiments were performed in accordance with Danish legislation on animal welfare and approved by the Danish Council for Supervision with Experimental Animals (license 2012-15-2934-00739). Mice were housed under standard conditions, kept in a 12-hour light/dark cycle, had access to food and water ad libitum, and were provided with enrichment for improved care. Mice with any type of distress or illness were immediately euthanized by cervical dislocation. 


\section{In Vitro Force Measurements}

Immediately after excision, the muscles were placed in Krebs-Ringer bicarbonate solution that contained $122 \mathrm{mM}$ sodium chloride, $25 \mathrm{mM}$ sodium bicarbonate, $2.8 \mathrm{mM}$ potassium chloride, $1.2 \mathrm{mM}$ monopotassium phosphate, $1.2 \mathrm{mM}$ magnesium sulfate, $1.3 \mathrm{mM}$ calcium chloride, and $5 \mathrm{mM}$ D-glucose. A small loop of surgical silk was tied to each tendon of the muscle, and the muscles were mounted vertically between a fixed hook and an isometric force transducer (K30 type 351; Hugo Sachs Elektronick, MarchHugstetten, Germany) in a temperature-controlled chamber at $30^{\circ} \mathrm{C}$ (Schuler Organbad, Hugo Sachs Elektronick) and gassed with a mixture of $95 \%$ oxygen and $5 \%$ carbon dioxide ( $\mathrm{pH}$ 7.4) throughout the experiments. Muscles were gently adjusted to the length eliciting maximum force with a single-twitch stimulus, equilibrated for 30 minutes before stimulation with a test stimulus $(60 \mathrm{~Hz}, 0.2$-millisecond duration for 1.5 seconds). To test the muscle forcefrequency relationship, a series of stimulations at 10, 20, $30,40,60,80,100,150,200$, and $250 \mathrm{~Hz}$ for 1.5 seconds were elicited, with 1-minute rest in between. After a 5-minute rest, the muscles were subjected to a 3-minute fatigue protocol in which contractions were evoked by $100 \mathrm{~Hz}$ on a duty cycle of 400 milliseconds every 5 seconds (EDL) or $60 \mathrm{~Hz}$ on a duty cycle of 500 milliseconds every 3 seconds (soleus). To test muscle contractile properties after the fatigue protocol, test stimuli were elicited after 1-, $10-$, and 30-minute recovery.

\section{SPARC Knockdown Cells}

In the C2C12 myoblast cell line (CRL-1772, LGC Promochem, ATCC, Manassas, VA), SPARC protein was knocked down using the BLOCK-iT expression system (Life Technologies, Carlsbad, CA) according to the manufacturers' instruction. Predesigned DNA oligos (Sparc BLOCK-IT miR RNAi select, Life Technologies) were used. In brief, the single-stranded DNA oligos were annealed and ligated with the linearized pcDNA6.6-GW/ EmGFP-miR vector followed by transformation into competent TOP10 Escherichia coli. Resulting clones were sequenced and plasmids were purified and transfected into C2C12 cells using Lipofectamine 2000 Reagent (Life Technologies) according to the manufacturers' instruction. Clones were screened for expression of Emerald Green Fluorescent Protein, and a stable cell line was established by selective growth using Blasticidine $(5 \mu \mathrm{g} / \mathrm{mL})$.

\section{C2C12 Culture}

$\mathrm{C} 2 \mathrm{C} 12$ and $\mathrm{C} 2 \mathrm{C} 12$ SPARC knockdown cells ( $\left.22 \mathrm{C} 12^{\text {SPARC KD }}\right)$ were cultured at $37^{\circ} \mathrm{C}$ with $5 \%$ carbon dioxide in a humidified chamber. For proliferation, cells were grown in Dulbecco's modified Eagle's medium with high glucose (Life Technologies) supplemented with $10 \%$ fetal bovine serum and antibiotics (50 U/mL of penicillin and $50 \mu \mathrm{g} / \mathrm{mL}$ of streptomycin); in addition, $\mathrm{C} 2 \mathrm{C} 12^{S P A R C} K D$ cells were continuously grown with $5 \mu \mathrm{g} / \mathrm{mL}$ of Blasticidine in the medium.

To induce myofiber formation (differentiation), cells were cultured to approximately $90 \%$ confluence in proliferation media then changed to Dulbecco's modified Eagle's medium with high glucose supplemented with $2 \%$ fetal bovine serum and antibiotics.

\section{Human Samples}

Biopsy specimens for isolation of primary myoblasts were obtained from voluntary participants after they gave written informed consent. The Regional Scientific Ethical Committee for Southern Denmark (S-20070079) approved the use of the biopsy specimens. Biopsy specimens from dystrophic patients were obtained from the archive at the Department of Clinical Pathology, Odense University Hospital, Odense, Denmark. The use of human archival tissue for protein expression analyses during this study was approved by The Regional Scientific Ethical Committee for Southern Denmark (20070075).

\section{Primary Human Myoblast Culture}

The primary human myoblast culture was established from a muscle biopsy specimen taken from the vastus lateralis as described previously. ${ }^{29}$ The isolated cells were grown (maximum of 7 passages) in extracellular matrix (SigmaAldrich, Copenhagen, Denmark)-coated T75 flasks (Nunclon, VWR, Radnor, PA) in growth medium that consists of Dulbecco's modified Eagle's medium supplemented with $10 \%$ fetal bovine serum and antibiotics.

\section{Real-Time Quantitative PCR}

Total RNA was extracted from $\mathrm{C} 2 \mathrm{C} 12$ and $\mathrm{C} 2 \mathrm{C} 12^{\text {SPARC KD }}$ cells using TRIzol (Life Technologies), quantified using the NanoDrop Spectrophotometer ND-1000 and 500 ng reverse transcribed to cDNA using the High-Capacity cDNA Archive Kit (Applied Biosystems, Foster City, CA) in a total volume of $100 \mu \mathrm{L}$. TaqMan Gene Expression Assays (Applied Biosystems) were used to analyze Sparc and the reference genes Hprtl and Tfrcl. The real-time quantitative PCR reactions were run in triplicates on duplicate samples using the ABI PRISM 7900 HT Real Time PCR System and analyzed using SDS software version 2.1 (Applied Biosystems) and qBase software. ${ }^{30}$

\section{Tissue Preparation}

Formalin-fixed, paraffin-embedded mouse (TA from wt and $m d x$ mice) and human muscle tissue as well as mouse tissue (TA from the regeneration study, EDL and soleus muscle) fixated in $4 \%$ normal buffered formaldehyde (TA only), embedded in Tissuetek, and frozen in nitrogen cooled 
isopentane was cut in 4- to 5- $\mu \mathrm{m}$ sections and stained with hematoxylin and eosin for morphologic analysis and Sirius red for analysis of fibrosis. For immunohistochemical or Duolink analysis, heat-induced antigen retrieval was performed on paraffin-embedded sections by boiling the sections in Tris-EGTA buffer ( $\mathrm{pH}$ 9.0) for the first 15 minutes at $900 \mathrm{~W}$ then for 9 minutes at $440 \mathrm{~W}$. The sections were allowed to cool in the buffer before the staining protocol was continued. All sections, paraffin and cryosections, were blocked for endogenous peroxidase and biotin activity.

\section{Immunohistochemistry}

Human tissue sections were incubated with mouseanti-human SPARC (ncl-o-nectin; Novocastra, Wetzlar, Germany) 1:50 with rabbit-anti-human Hsp27 (Spa-803; Stressgen, Victoria, British Columbia, Canada) 1:2000 and detected using Alexa flour 555 goat-anti-rabbit IgG (Life Technologies) 1:200 and Alexa fluor 488 goat-anti-mouse IgG 1:200 (Life Technologies).

Mouse tissue sections from paraffin-embedded tissue were incubated with rat-anti-mouse SPARC (R\&D Systems, Minneapolis, MN) 1:500, mouse-anti-rat myogenin (F5D, Dako Cytomation, Carpinteria, CA) 1:200, or rat-anti-mouse CD45 [30-F11 (Ly 5); BD Pharmingen, San Jose, CA] 1:50 and detected using biotinylated rabbit-antirat IgG (Dako Cytomation) 1:200 with streptavidin-horseradish peroxidase (HRP) (Dako Cytomation) or the ARK kit (K3954, Dako Cytomation) and $\mathrm{DAB}^{+}$as chromogen (Dako Cytomation). Nuclei were counterstained using Mayer's hemalum with $4.5 \%$ chloralhydrate.

Frozen mouse tissue sections (TA regeneration study) was fixated in $4 \%$ normal buffered formaldehyde and incubated with rat-anti-mouse CD45 [30-F11 (Ly 5); BD Pharmingen] 1:100, mouse-anti-human desmin (Dako Cytomation, M0760) 1:50, or mouse-anti-rat myogenin (F5D, Dako Cytomation) 1:200. The CD45 reaction was detected using rabbit-anti-rat antibody (Dako Cytomation) diluted 1:200 followed by ready-to-use anti-rabbit HRPlabeled polymer (EnVision+ System, Dako Cytomation), and the desmin and myogenin reactions were detected using the ARK kit. All reactions were counterstained as above.

For double fluorescence staining, mouse sections were incubated with rat-anti-mouse SPARC (R\&D Systems) 1:500 or 1:100.000 (for detection with tyramide-signal amplification) with rabbit-anti-human S100A4 (Abcam, Cambridge, United Kingdom) 1:200, rabbit-anti-chicken neural cell adhesion molecule (NCAM) (AB5032; Chemicon International, Billerica, MA) 1:1000, rat-anti-mouse F4/80 (macrophage) (AbD Serotec, Hercules, CA) 1:2000 overnight, rat-anti-mouse Ly-6G (BD Pharmingen) 1:1000 overnight, or rabbit-anti-rat Hsp27 (Cell Signaling Technology, Danvers, MA) 1:50. Secondary antibodies used were Alexa flour 555 goat-anti-rabbit IgG (Life Technologies) 1:200, Alexa fluor 488 or 555 goat-anti-rat (Life Technologies) 1:200, and Alexa fluor 488 goat-anti-mouse IgG (Life
Technologies) 1:200 were used. For analysis of SPARC with Ly6G or F4/80, antibody reactions were performed sequentially, including a denaturing step where sections were boiled in TEG buffer between reactions to avoid cross-reactions of the two secondary antibody steps. For double staining with Ly-6G, the SPARC antibody reaction was detected using Tyramide-Alexa 488 1:50 (Life Technologies), and for analysis of SPARC with F4/80, the F4/80 antibody reaction was detected with Tyramide-Alexa 488 1:50.

All fluorescence staining was mounted using Vectashield with DAPI (Vector Laboratories Inc, Burlingame, CA).

For fiber-type determination, frozen mouse sections, either fixated before freezing (TA contralateral and day 7 after injury) or fresh frozen (EDL and soleus), were brought to room temperature and then placed in $95^{\circ} \mathrm{C}$ Tris-EGTA buffer, $\mathrm{pH}$ 9.0, for 15 minutes, followed by blocking for endogenous biotin and peroxidase. Sections were incubated with $2 \%$ bovine serum albumin (BSA) or Tris-buffered saline (TBS) for 10 minutes followed by either myosin heavy chain (MHC) fast (MY32, Sigma-Aldrich) 1:1000 or MHC slow (MEDCLA67-1, Accurate Chemicals, Westbury, NY) 1:25 for 30 minutes. The reactions were detected using the ARK kit (K3954, Dako Cytomation) according to standard protocol with $\mathrm{DAB}^{+}$as chromogen. Nuclei were counterstained as above.

\section{Image Acquisition and Data Preparation}

Epifluorescent images were obtained with a Leica DM LB2 microscope and the digital camera Leica DFC 300FX (Leica, Wetzlar, Germany). Images were acquired with the Leica Application Suite software version 3 at identical settings to allow a direct comparison and analyzed as TIFF files using Adobe Photoshop CS5 software version 12 (Adobe, San Jose, CA). Images were used either directly with changes in size and resolution (from 300 to $600 \mathrm{dpi}$ ) only or equally improved in all channels by using the image tab followed by "adjustments," and then using automatic tone or color, light or contrast, or shadow or highlight changes. Scans of Western blot films were imported in 1000-dpi resolution, changed to gray scale, and improved using light or contrast changes. The Leica epifluorescent objectives [HC/HCX (Harmonic Compound System, $\mathrm{X}$ indicates compatibility with past optics) plan APO (Apochromats)] used in the study had the following numerical apertures: $20 \times / 0.70,63 \times / 1.40$ to 0.60 oil and $100 \times / 1.40$ to 0.70 oil. The filter cubes used for visualizing fluorescent protein staining had the following specifications: L5; excitation filter (EF) BP480/40, dichromatic mirror (DM) 505, suppression filter (SF) BP 527/30, N2.1 (equivalent to Texas Red); EF BP 562/40, DM LP 593, SF BP 624/40, A4 (equivalent to DAPI); EF BP 360/40, DM 400, SF BP 470/40 and Y3; EF BP 545/40, DM 565, SF BP $610 / 75$.

Electron microscopic images were obtained using the JEM 1400 Plus transmission electron microscope (JEOL, 
Freising, Germany) and the bottom-mounted digital camera Quemesa (Olympus, Southend-on-Sea, United Kingdom). Images were acquired using iTEM software Olympus 2010 version 5.2 (Build 3554) and analyzed as JPEG files using Adobe Photoshop CS5 software.

\section{Analysis of Necrosis}

The presence of necrosis after regeneration was analyzed by incubating frozen mouse muscle sections with a 1:200 solution of donkey anti-mouse IgG (Alexa Flour 555, Life Technologies) and mounting the sections with DAPI (Vectashield, Vector Laboratories Inc). Analysis was performed using the program VIS version 4.5.6.440 (Visiopharm, Hoersholm, Denmark) and a fluorescence microscope (Leica DM6000B) coupled to a computer and a camera (Olympus DP72). For this analysis, a $20 \times$ objective and the filter cubes Y3 and DAPI were used (see specifications above). Measurements of the entire cross-sectional area and the necrotic area were performed automatically. The microscope slides were placed in the microscope ( 8 at the same time), then fluorescence filters (Y3, DAPI) were chosen and focus and exposure time fine-tuned and saved. Then the microscope was briefly changed to bright field and a small magnification, after which a super image was obtained for every tissue section on the 8 microscope slides. This allowed for marking the entire tissue section on the computer screen using the super images. The microscope was changed back to the fluorescence filters and the chosen settings, and at $\times 20$ magnification images were obtained to cover each entire tissue section and compiled automatically into one image showing both fluorophores at the same time. With the use of the VIS software, the whole region was measured by manually drawing around the tissue section, and by the same method, the area of interest (here necrosis) was marked in a similar way, allowing for quantification of necrosis per area.

\section{Morphometrics}

The number of cells expressing SPARC, myogenin, and CD45 protein in $m d x$ and wt mice and the number of cells expressing CD45 or myogenin protein in regenerating muscle was estimated on sections ( $n=4$ to 5 mice) by counting cells using a microscope (Olympus BX50) equipped with a camera (Olympus U-PMTVC) connected to a motorized cross board and a computer. The CAST software version 2.1.6.0 (Olympus Danmark A/S, Ballerup, Denmark) was used to systematically select fields to include the entire muscle section. A cross-sectional area was measured by drawing around the area of interest (entire section or regenerating area) by means of the computer screen and the CAST software. CAST was then able to calculate the size of the area, and this measurement was used for normalization of counts.

For analysis of fiber-type composition (fast or slow) and fiber diameter, CAST was used to count and measure a minimum of 100 individual fibers for each fiber type on each section (TA, EDL, and soleus muscles). The magnification used was $\times 10$, and the entire section was included as counting area. Diameters were measured by manually drawing transverse lines across all fibers.

The objectives used had the following numerical apertures: Uplan/APO $10 \times / 0.40$ and Uplan/APO $20 \times / 0.70$.

\section{Immunocytochemistry}

$\mathrm{C} 2 \mathrm{C} 12$ and $\mathrm{C} 2 \mathrm{C} 12^{\text {SPARC KD }}$ cells grown on glass coverslips were fixated in $4 \%$ normal buffered formaldehyde, permeabilized in $0.5 \%$ Triton-X, and blocked with $2 \%$ BSA/ TBS. The samples were incubated with the following primary antibodies: rat-anti-mouse SPARC (R\&D Systems) 1:500, rabbit-anti-actin (all isoforms) (1844-1, Epitomics, Burlingame, CA) 1:250, mouse-anti-rabbit $\alpha$-actin (sc-58671, Santa Cruz Biotechnology, Santa Cruz, CA) 1:100, or mouse-anti-bovine $\gamma$-actin (sc-65638, Santa Cruz Biotechnology) 1:100 for 1 hour. For staining of $\beta$-actin, cells were fixated in $100 \%$ methanol for 5 minutes, permeabilized in $0.5 \%$ Triton- $X$, and blocked with $2 \%$ BSA/ TBS. Cells were incubated with rabbit-anti-human $\beta$-actin (ab8227, Abcam) overnight at $4^{\circ} \mathrm{C}$. After incubation with primary antibody, samples were washed in Tris-buffered saline with Tween 20 before incubation with the secondary antibodies goat-anti-mouse-Alexa 488 or Alexa 555 1:200, goat-anti-rat-Alexa 555 1:200 or goat-antirabbit-Alexa 555 1:200 (Life Technologies) for 1 hour, after which they were mounted with SlowFade Diamond Antifade Mountant with DAPI (Life Technologies).

For staining of human myoblasts, cells were treated as above and incubated with mouse-anti-human SPARC (nclo-nectin; Novocastra) 1:50 and rabbit-anti-human Hsp27 (Spa-803; Stressgen) 1:300 followed by Alexa flour 555 goat-anti-rabbit IgG (Life Technologies) 1:200 and Alexa fluor 488 goat-anti-mouse IgG 1:200 (Life Technologies) and mounted with SlowFade Diamond Antifade Mountant with DAPI (Life Technologies).

\section{Duolink Interaction Studies}

Specific protein-protein interactions between SPARC and Hsp27 or SPARC and actin were analyzed using Duolink in situ reagents with proximity ligation assay probes (Olink Bioscience, Uppsala, Sweden) on human primary myoblasts and human muscle tissue. Human myoblasts were fixated in $4 \%$ formaldehyde and permeabilized with $0.5 \%$ Triton $\mathrm{X}$ followed by incubation with Duolink blocking solution in a preheated humidified chamber at $37^{\circ} \mathrm{C}$ for 30 minutes. Primary antibody solution containing mouse-anti-human SPARC (ncl-o-nectin; Novocastra) 1:50/rabbit-anti-human Hsp27 (SPA-803; Stressgen) 1:2000 or mouse-anti-human SPARC (ncl-o-nectin; Novocastra) 1:50/rabbit-anti-human actin (1844-1; Epitomics) 1:250 was added for 1 hour at room temperature. 
Tissue sections were treated as described, and then the sections were incubated with Duolink blocking solution followed by primary antibody solution containing mouse-anti-human SPARC (ncl-o-nectin; Novocastra) 1:25/rabbit-anti-human actin (1844-1; Epitomics) 1:20 as above. Then, cells and sections were incubated with proximity ligation assay probes PLUS and MINUS diluted $1: 5$ for 1 hour at $37^{\circ} \mathrm{C}$, followed by incubation with ligation-ligase solution (Duolink) for 30 minutes at $37^{\circ} \mathrm{C}$. After ligation, the samples were incubated with an amplification polymerase solution for 100 minutes at $37^{\circ} \mathrm{C}$.

Myoblasts was counterstained for 1 hour at room temperature with Vimentin Dylight 488 (Epitomics) diluted 1:100. Tissue sections were counterstained for NCAM by blocking with $2 \% \mathrm{BSA} / \mathrm{TBS}$ and then incubating with mouse-anti-human NCAM (ms-1149-p; Thermo Fisher Scientific, Waltham, MA) 1:25 for 45 minutes at room temperature followed by Alexa flour 488 goat-anti-mouse IgG (Life Technologies) 1:200 for 45 minutes at room temperature. All samples were mounted with Mounting medium (Duolink) containing DAPI before microscopic analysis.

\section{Co-Immunoprecipitation and Mass Spectrometry}

Magnetic beads (Dynabeads, Life Technologies) were coupled with $5 \mu \mathrm{g} / \mathrm{mg}$ of SPARC antibody (rat-antimouse SPARC IgG2B, R\&D Systems) on a roller overnight at room temperature. Beads incubated with vehicle only were used as control. Protein was extracted from differentiated $\mathrm{C} 2 \mathrm{C} 12$ and $\mathrm{C} 2 \mathrm{C} 12^{S P A R C} K D$ cell pellets by incubating the cell pellets in a 1:9 ratio with extraction buffer $(110 \mathrm{mmol} / \mathrm{L}$ potassium acetate, $0.5 \%$ Triton X-100, $2 \mathrm{nmol} / \mathrm{L}$ magnesium chloride, $1 \mathrm{mmol} / \mathrm{L}$ dithiothreitol, $100 \mathrm{mmol} / \mathrm{L}$ sodium chloride, $1 \mu \mathrm{L} / \mathrm{mL}$ of protease inhibitor, $\mathrm{pH}$ 7.4), followed by incubation on ice for 15 minutes and centrifugation at $2600 \times g$ for 5 minutes at $4^{\circ} \mathrm{C}$. The supernatants were used immediately for immunoprecipitation. Then $1.5-\mathrm{mg}$ antibody and control coupled beads were incubated with cell lysate and placed on a roller for 30 minutes at $4^{\circ} \mathrm{C}$. Beads were magnetically removed and washed, and then the immunoprecipitation products were eluted and run on a $4 \%$ to $12 \%$ Bis-Tris SDS-PAGE gel for protein separation under reducing conditions. The gel was silver stained according to the standard protocol, and relevant protein bands (present in $\mathrm{C} 2 \mathrm{C} 12$ but absent from $\mathrm{C} 2 \mathrm{C} 12^{S P A R C} K D$ ) were excised from one-dimensional -gel and subsequently in-gel digested. Briefly described, the gel pieces were washed twice in $50 \mathrm{mmol} / \mathrm{L}$ ammonium bicarbonate and $50 \%$ ethanol and then dehydrated in ethanol before reduction with $10 \mathrm{mmol} / \mathrm{L}$ dithiothreitol and alkylation with $50 \mathrm{mmol} / \mathrm{L}$ iodoacetamide in $50 \mathrm{mmol} / \mathrm{L}$ ammonium bicarbonate. The alkylated gel pieces were washed and dehydrated as above and digested with trypsin overnight at $37^{\circ} \mathrm{C}$ followed by acidification with $1 \%$ trifluoroacetic acid. The tryptic peptides were desalted using the reverse phase microtip purification technique and subsequently eluted onto Bruker UltraFlex polished steel target. Peptide mass fingerprints (PMFs) and MALDI-TOF/TOF spectra were obtained on an UltraFlex matrix-assisted laser desorption/ionization tandem time-of-flight (MALDI-TOF/TOF) instrument (Bruker Daltonics, Billerica, MA). All PMFs were three points externally calibrated using known masses of tryptic peptides and annotated by the flex analysis software version 3.4 (Bruker Daltonics). The data were searched against an in-house NCBInr database (ftp://ftp.ncbi.nlm. nih.gov/refseq/release/complete, release 53, last accessed June 28, 2012) using an in-house MASCOT server (Matrix Science, Columbia, SC). Peptide tolerance was set to 80 ppm for PMFs and 0.9 Da for MALDI-TOF/TOF spectra. Cysteine carbamidomylation was set as fixed modification. Methionine oxidation was set as variable modification. One missing cleavage was allowed per peptide. For positive protein identification, the score of the result of $(-10 \times$ $\log (p))$ had to be over the significance threshold level $(P=0.05)$.

\section{Western Blot}

Cell samples were washed twice in phosphate-buffered saline. Total protein was extracted by resuspending cell pellets in RIPA Lysis and Extraction Buffer (Themo Fisher Scientific) containing $1 \times$ Halt Protease Inhibitor Cocktail (Thermo Fisher Scientific) and $1 \times$ Halt phosphatase Inhibitor Cocktail (Thermo Fisher Scientific) followed by incubation on ice for 30 minutes. The lysate was centrifuged $\left(15\right.$ minutes, $\left.12,000 \times g, 4^{\circ} \mathrm{C}\right)$ and the supernatant was stored at $-80^{\circ} \mathrm{C}$ until use. Protein concentration was determined using Pierce BCA protein assay kit (VWR).

For analysis, approximately $10 \mu \mathrm{g}$ of protein was added to the loading buffer (Thermo Fisher Scientific) and sample reducing agent (Thermo Fisher Scientific), heated to $95^{\circ} \mathrm{C}$ for 5 minutes, and loaded onto $4 \%$ to $12 \%$ Bis-Tris gels (Thermo Fisher Scientific). The gel was run using $1 \times$ MES buffer (Thermo Fisher Scientific) for 1 hour at $120 \mathrm{~V}$ constant and electroblotted unto $0.45-\mu \mathrm{m}$ polyvinylidene difluoride membranes (Millipore, Billerica, MA). Transfer and equal loading were validated using a Ponceau $S$ staining of the membranes after transfer. The membranes were blocked with either 5\% skimmed milk and Tris-buffered saline with Tween 20 or 5\% BSA and Trisbuffered saline with Tween 20 for 1 hour at room temperature and incubated overnight at $4{ }^{\circ} \mathrm{C}$ with the following primary antibodies: rat-anti-mouse SPARC $(1: 1000, \mathrm{R} \& \mathrm{D}$ Systems), rabbit-anti-human $\beta$-actin (1:1000, ab8227, Abcam), mouse-anti-rabbit $\alpha$-actin (1:200, sc-58671, Santa Cruz Biotechnology), mouse-anti-bovine $\gamma$-actin (1:2000, sc-65638, Santa Cruz Biotechnology), rabbit-anti-human vinculin $(1: 1000,4650$, Cell Signaling Technology), rabbit-anti-human RhoA (1:1000, no. 2117, 
Cell Signaling Technology), rabbit-anti-human glyceraldehyde-3-phosphate dehydrogenase (1:1000, sc-25778, Santa Cruz Biotechnology). The following day blots were washed in Tris-buffered saline with Tween $20(3 \times)$ and incubated for 1 hour at room temperature with goatanti-rabbit-HRP (1:1000, p0448, Dako Cytomation), goat-anti-mouse-HRP (1:1000, p0447, Dato Cytomation), or goat-anti-rat-HRP (1:5000, A10549, Life
A

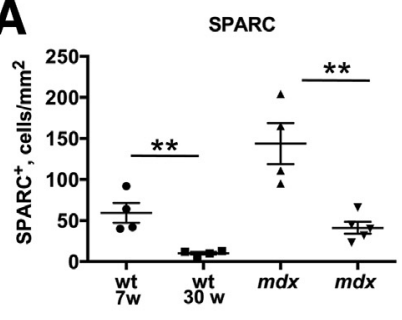

D

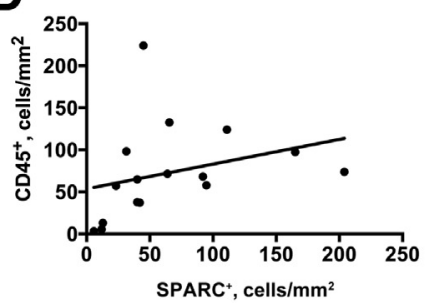

F
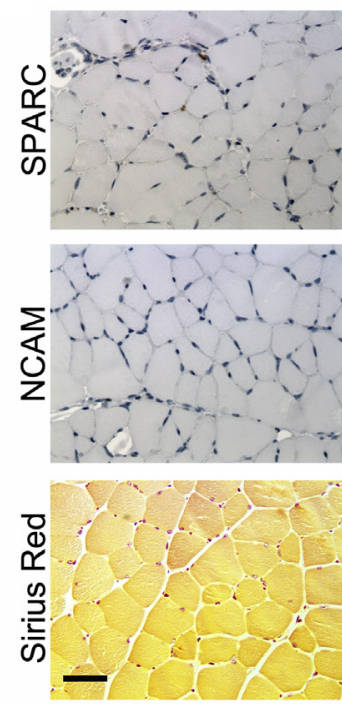

wt 7-10 weeks
B

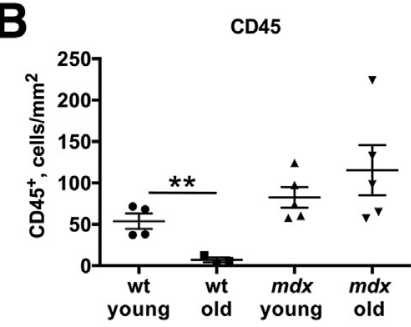

E

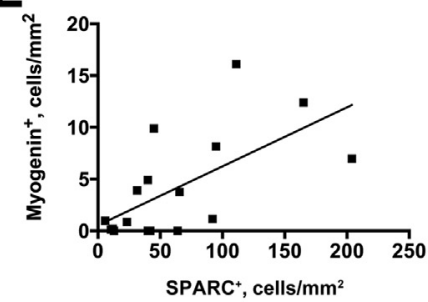

C

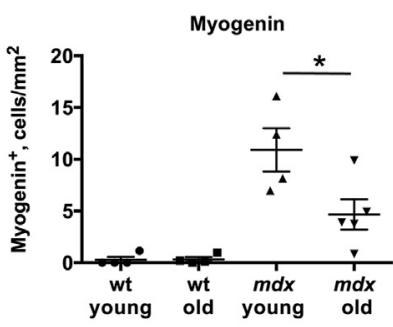

G
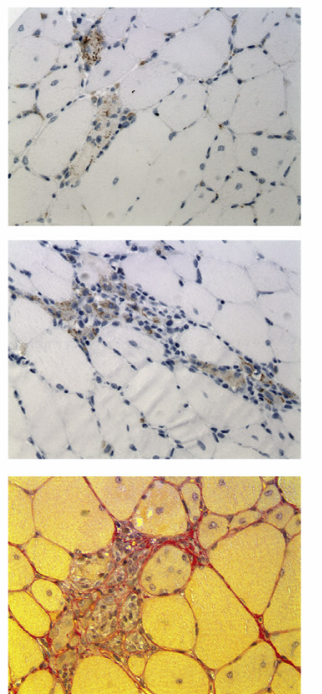

$m d x$ 7-10 weeks

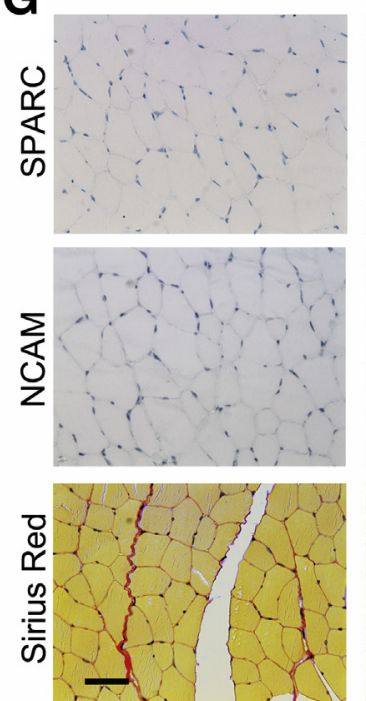

wt 24-30 weeks
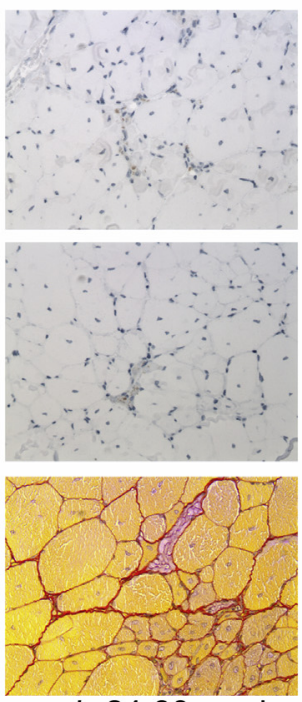

mdx 24-30 weeks

Figure 1 Secreted protein acidic and rich in cysteine (SPARC) expression correlates with active regeneration. Morphometric analysis of cells expressing SPARC (A), CD45 (B), and myogenin (C) in 7- to 10-week-old (young) and 24- to 30-week-old (older adults) dystrophic ( $m d x)$ and wild-type (wt) mice. The number of cells expressing SPARC decreased significantly with age in both wt ( 7 to 10 weeks: $59.5 \pm 12$ versus 24 to 30 weeks: $10.3 \pm 1.57, P<0.01$, unpaired $t$-test) and $m d x$ ( 7 to 10 weeks: $144 \pm 25$ versus 24 to 30 weeks: $41 \pm 7.1, P<0.01$, unpaired $t$-test) mice. Myogenin-positive cells were mostly absent from wt mice regardless of age ( 7 to 10 weeks: $0.29 \pm 0.29$ versus 24 to 30 weeks: $0.33 \pm 0.22, P>0.05$, unpaired $t$-test), whereas the number of myogenin-positive cells was reduced in 24 - to 30 -week-old $m d x$ mice compared with 7 - to 10 -week-old $m d x$ mice ( 7 to 10 weeks: $10.9 \pm 2.1$ versus 24 to 30 weeks: $4.7 \pm 1.5, P<0.05$, unpaired $t$-test). Few CD45-positive cells were observed in both 7- to 10-week-old and 24- to 30-week-old wt mice; still the number was reduced with age (wt: 7 to 10 weeks: $53.7 \pm 9.3$ versus 24 to 30 weeks: $7.2 \pm 2.9, P<0.01$ ), whereas in the $m d x$ mice, the number of leukocytes remained high, both in the 7- to 10-week-old group and the 24- to 30-week-old group (7 to 10 weeks: $82.7 \pm 12.5$ versus 24 to 30 weeks: $115.4 \pm 30.3$, $P>0.05$, unpaired $t$-test). Correlation analysis between SPARC and CD45 (D) as well as SPARC and myogenin (E) revealed a direct correlation between SPARC and myogenin $(P<0.01$, Pearson correlation) but there was no correlation between SPARC and CD45 $(P>0.05$, Pearson correlation). F: Representative images of SPARC, neural cell adhesion molecule (NCAM) and Sirius red staining in young (7- to 10-week-old) wt and $m d x$ mice. The figure depicts the SPARC staining used for quantification in $\mathbf{A}$, and NCAM reveals active regeneration in 7 to 10 -week-old $m d x$ mice. Sirius red is a fibrosis marker revealing beginning thickening of connective tissue in the young $m d x$ mice. G: Representative images of SPARC, NCAM and Sirius red staining in older adult (24- to 30-week-old) wt and $m d x$ mice. The figure depicts the SPARC staining used for quantification in A, and NCAM reveals that active regeneration is barely present in the 24- to 30-week-old $m d x$ mice. Sirius red reveals increased thickening of connective tissue in the 24 - to 30 -week-old $m d x$ mice. Scale bar $=50 \mu m(\mathbf{F}$ and $\mathbf{G})$. 

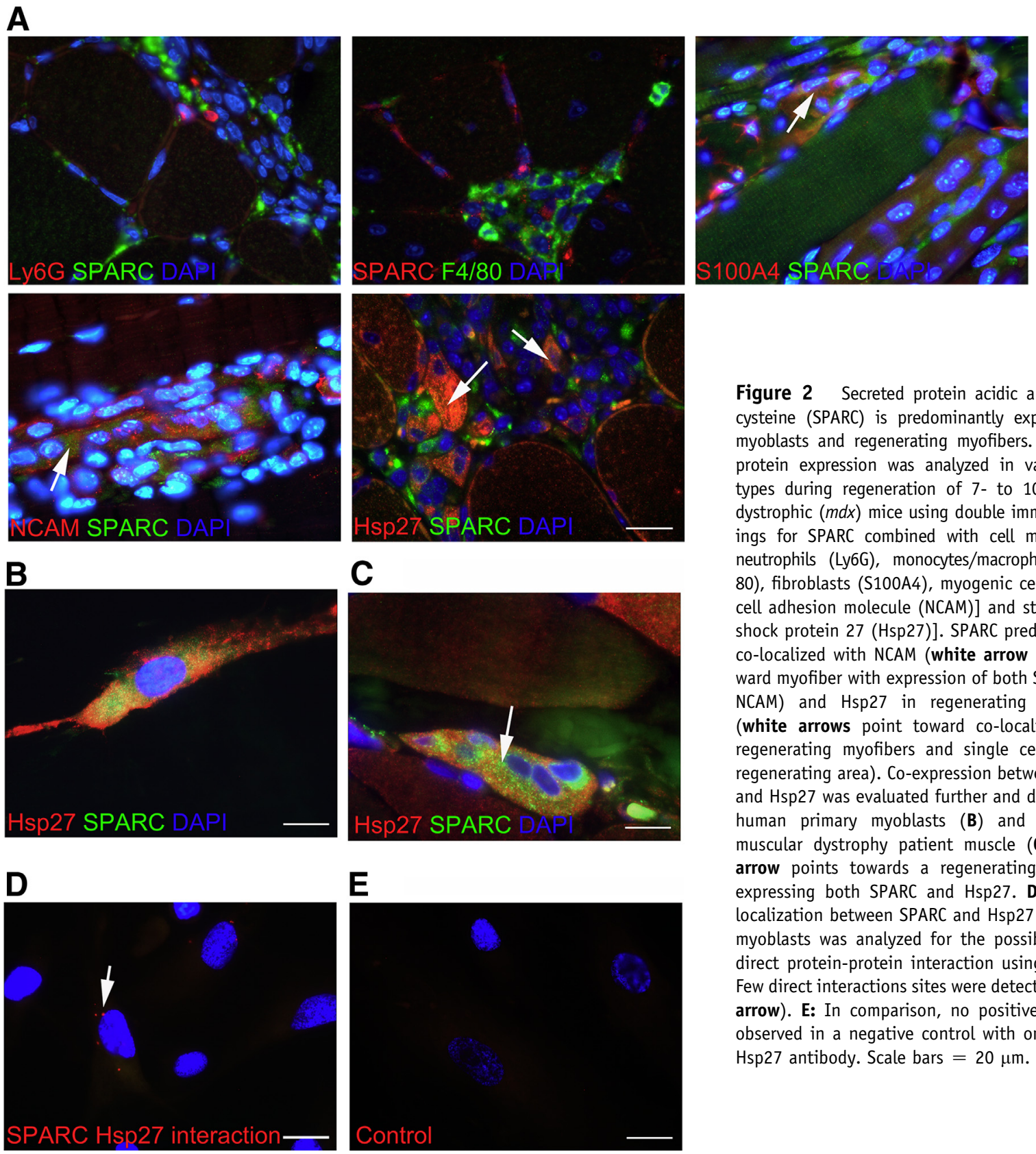

Figure 2 Secreted protein acidic and rich in cysteine (SPARC) is predominantly expressed by myoblasts and regenerating myofibers. A: SPARC protein expression was analyzed in various cell types during regeneration of 7- to 10-week-old dystrophic $(m d x)$ mice using double immunostainings for SPARC combined with cell markers for neutrophils (Ly6G), monocytes/macrophages (F4/ $80)$, fibroblasts (S100A4), myogenic cells [neural cell adhesion molecule (NCAM)] and stress [heat shock protein 27 (Hsp27)]. SPARC predominantly co-localized with NCAM (white arrow points toward myofiber with expression of both SPARC and NCAM) and Hsp27 in regenerating myofibers (white arrows point toward co-localization in regenerating myofibers and single cells in the regenerating area). Co-expression between SPARC and Hsp27 was evaluated further and detected in human primary myoblasts (B) and Duchenne muscular dystrophy patient muscle (C). White arrow points towards a regenerating myofiber expressing both SPARC and Hsp27. D: The colocalization between SPARC and Hsp27 in human myoblasts was analyzed for the possibility of a direct protein-protein interaction using Duolink. Few direct interactions sites were detected (white arrow). E: In comparison, no positive signal is observed in a negative control with omission of Hsp27 antibody. Scale bars $=20 \mu \mathrm{m}$.

Technologies), washed in Tris-buffered saline with Tween $20(3 \times)$ and developed using Novex ECL HRP Chemiluminescent Substrate kit (Life Technologies) and standard X-ray film. Glyceraldehyde-3-phosphate dehydrogenase was used as loading control.

\section{Transmission Electron Microscopy}

Muscle tissue (EDL and soleus) from $S P A R C^{+/+}$and $S_{P A R C}^{-1-}$ mice were fixated in $2 \%$ glutaraldehyde/ phosphate-buffered saline and stained with $1 \%$ osmiumtetraoxide/phosphate-buffered saline, dehydrated, and embedded in epon (epoxyresin). Ultrathin sections $(60 \mathrm{~nm})$ were cut on a Leica Ultracut Ultramicrotome and placed on a grid, contrasted using 3\% uranylacetate/water and lead citrate (Leica Ultrostain 2) and analyzed.

\section{Statistical Analysis}

The unpaired $t$-test, multiple $t$-test with statistical significance determined using the Holm-Sidak method, 2-way analysis of variance, and Pearson correlation analysis with $P<0.05$ as the significance level were performed using GraphPad Prism software version 6 (GraphPad Software Inc, San Diego, CA). 


\section{Results}

SPARC Expression Is Predominantly Associated with Myoblasts and Immature Myofibers

We know that SPARC is highly expressed by actively regenerating skeletal muscle tissue in both Pax7-positive cells and regenerating fibers. ${ }^{2}$ However, we do not know whether this expression relates to the regeneration process only or if SPARC is also present in, for example, fibroblasts and is implicated in fibrosis. To investigate this further, we analyzed SPARC protein expression in TA from dystrophic $(m d x)$ and control (wt) mice at a young age (7 to 10 weeks) and an older age (24 to 30 weeks). We observed that SPARC expression was prominent in young dystrophic mice, which have active, ongoing regeneration (NCAM) (Figure 1F) with the number of positive cells decreasing as the disease progressed in older dystrophic mice ( 7 to 10 weeks versus 24 to 30 weeks $m d x: P<0.01$ ) (Figure $1 \mathrm{~A}$ ), where the regeneration process is substituted for fibrosis (NCAM and Sirius red) (Figure 1G). Moreover, SPARC expression was reduced with age in wt mice as well ( 7 to 10 weeks versus 24 to 30 weeks wt: $P<0.01$ ) (Figure 1A), suggesting that SPARC generally decreased with age, as has previously been published, ${ }^{17}$ and this decrease was not countered by the more severe dystrophic state of the adult $m d x$ mice $^{31}$ as observed by increased fibrosis (Sirius red) (Figure 1, F and G), pronounced, continued inflammation (CD45) (Figure 1B), and decreased regeneration (Myogenin and NCAM) (Figure 1, C and G). The highest number of SPARC-positive cells was detected in young dystrophic mice, and generally SPARC expression correlated with the marker for actively differentiating myoblasts, myogenin $\left({ }^{* * *} P<0.01\right)$ (Figure $1 \mathrm{E}$ ), and not the common leukocyte marker, CD45 $(P>0.05)$ (Figure 1D), suggesting a direct relation between SPARC expression and muscle tissue repair. This finding was further substantiated by double immunostaining for cell-specific markers (fibroblasts: S100A4, activated satellite cells, myoblasts, and regenerating myofibers: NCAM, neutrophils: Ly6G, and macrophages: F4/80) and SPARC, which revealed that intracellular SPARC expression during regeneration is associated directly with the muscle cells and immature, newly formed myofibers and not other components of the regeneration process, for example, fibrosis and inflammation (Figure 2A).

\section{SPARC Protein Is Co-Expressed with the Small Hsp27 in Myoblasts and Regenerating Fibers}

Next we investigated if the presence of SPARC in muscle cells and regenerating myofibers correlated with cellular stress. We

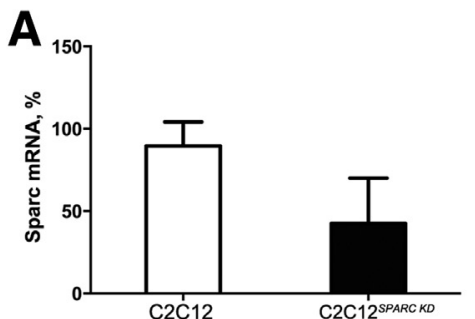

C

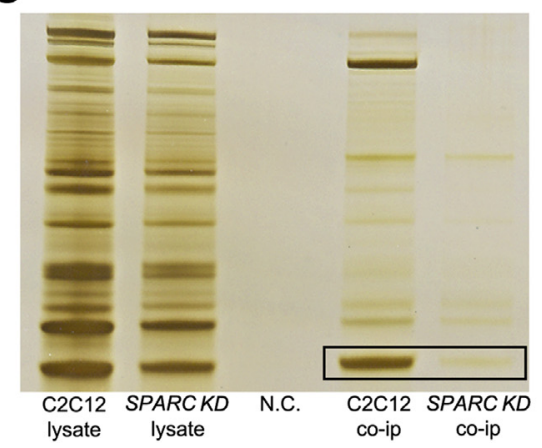

B

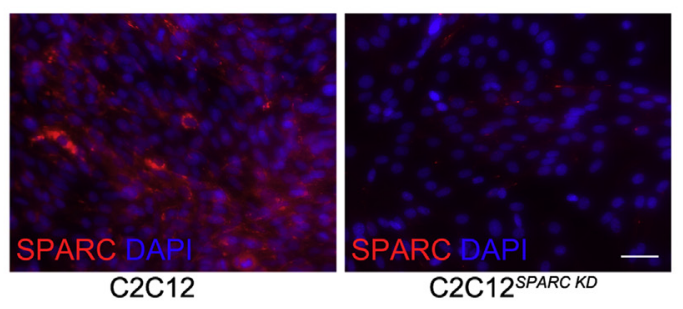

D

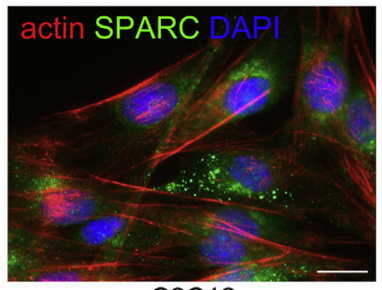

$\mathrm{C} 2 \mathrm{C} 12$

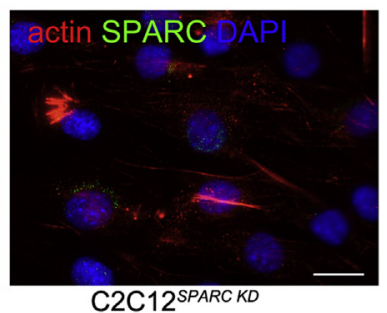

C2C12 $12^{\text {SPARC KD }}$

Figure 3 Identification of actin as a binding partner to secreted protein acidic and rich in cysteine (SPARC). A: To investigate the functional role of SPARC in vitro, SPARC was knocked down in $\mathrm{C} 2 \mathrm{C} 12$ cells $\left(C 2 C 12^{\text {SPARC KD }}\right.$ ) using shRNA. Using real-time quantitative PCR, we confirmed that Sparc expression was reduced on the mRNA level. B: Reduced expression of SPARC protein after knock down was observed using immunocytochemistry. We wanted to detect whether possible intracellular binding partner(s) to SPARC exist, and we therefore performed mass spectrometry analysis of proteins co-immunoprecipitating with SPARC. C: Silver staining of the gel used in identification of the interaction between SPARC and actin. The band identified as actin is enclosed by the black box. The gel visualizes the band spectra present in whole cell lysates from $\mathrm{C} 2 \mathrm{C} 12$ and $\mathrm{C}_{2} \mathrm{C} 12^{\text {SPARC } K D}$ cells, a negative control (N.C.) from immunoprecipitation with naked (vehicle-treated) beads from $\mathrm{C} 2 \mathrm{C} 12$ control cells and with SPARC antibody-coupled beads from $\mathrm{C} 2 \mathrm{C} 12$ and $\mathrm{C} 2 \mathrm{C} 12^{\text {SPARC } K D}$ cells. Cell samples used for immunoprecipitation were from differentiating cells. D: Immunocytochemical staining for localization and presence of SPARC (green) and actin (red) in C2C12 and C2C12 ${ }^{\text {PPARC KD }}$ proliferating cells. Expression of both proteins is clearly reduced in C2C12 $2^{\text {SPARC } K D}$. Data are expressed as means \pm SEM (A). Scale bars: $50 \mu \mathrm{m}$ (B); $20 \mu \mathrm{m}$ (D). co-ip, co-immunoprecipitation. 

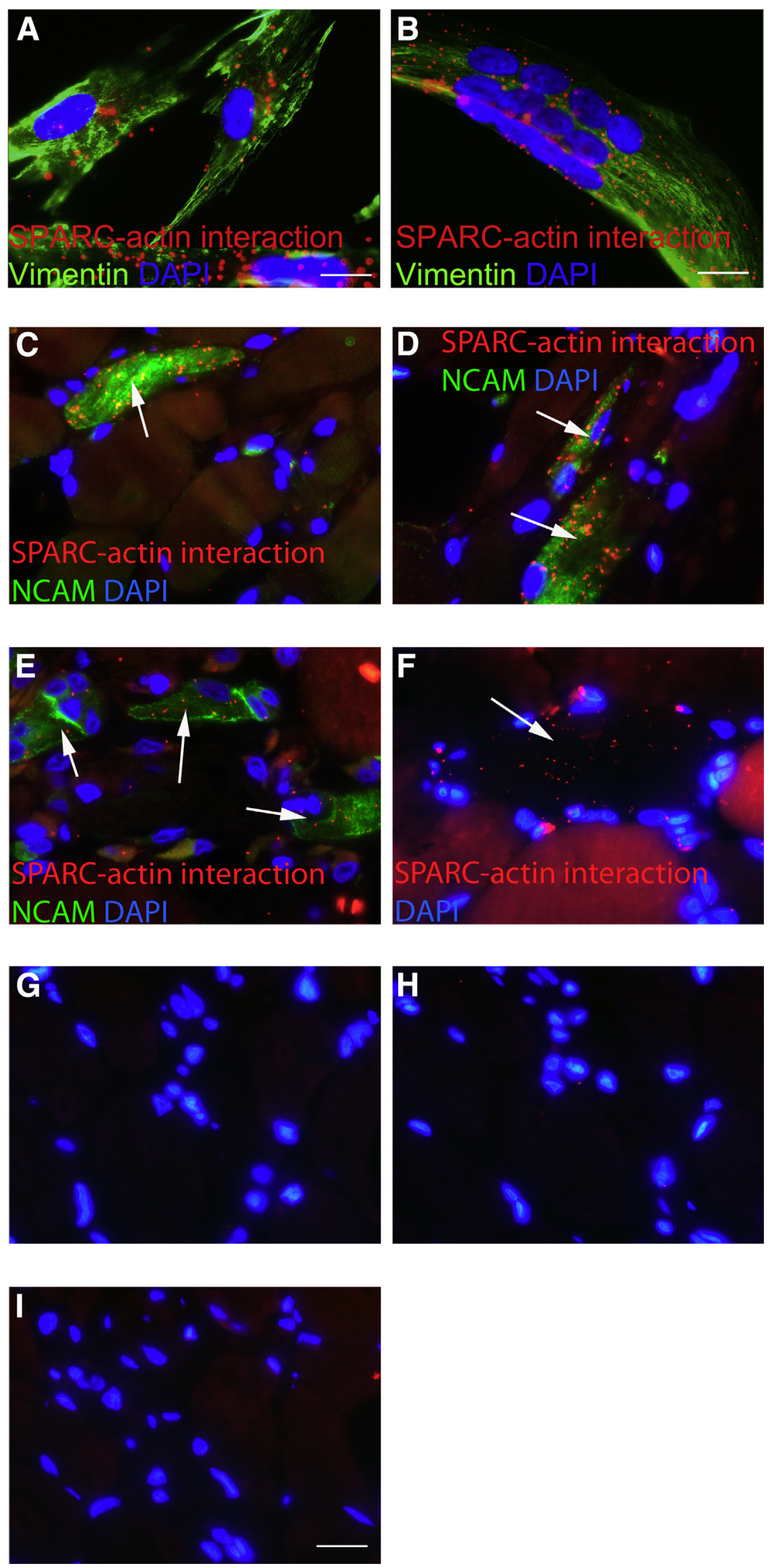

Figure 4 The interaction between actin and secreted protein acidic and rich in cysteine (SPARC) is present during proliferation and differentiation in vitro and in regenerating myofibers of dystrophic/myopathic patients in vivo. The interaction between actin and SPARC was analyzed in human muscle cells in vitro and in vivo in patients with muscle disorders all causing muscle damage using the Duolink proximity ligation assay. Red dots are equivalent to specific Duolink interactions, and each dot represents one interaction between actin and SPARC. Green indicates the vimentin (cells) or neural cell adhesion molecule (NCAM) (tissue) counterstaining, and blue is DAPI expression in nuclei. SPARC and actin is expressed and interacts in proliferating primary human satellite cells (A) and in myotubes derived from differentiating primary human satellite cells (B). The interactions are distributed throughout the cells both during proliferation and differentiation. C and D: Two different patients with Duchenne muscular dystrophy have a prominent number of interactions in regenerating myofibers (white arrows). E: A patient with compartment syndrome in the leg has the same interactions in regenerating myofibers (white arrows) (F) as does a patient with polymyositis (white arrow, this patient's biopsy specimen has not been counterstained with NCAM). G: A control staining for the interaction with omission of the actin antibody. H: A control staining for the interaction with omission of the SPARC antibody. I: A control staining for the interaction with omission of both primary antibodies. Scale bars $=20 \mu \mathrm{m}(\mathbf{A}-\mathbf{I})$. 
detected co-expression of SPARC with the small heat shock molecule Hsp27 in human myoblasts (Figure 2B) and in regenerating myofibers of both $m d x$ mouse muscle (Figure 2A) and Duchenne muscular dystrophy patient muscle (Figure 2C). Using Duolink, we analyzed whether the co-localization of SPARC and Hsp27 could be associated with a direct interaction between the two proteins. We only observed few sites of close proximity in human myoblasts, indicating no direct interaction between SPARC and Hsp27 (Figure 2, D and E).

\section{Identification of Actin as a Binding Partner to SPARC}

Next, we wanted to identify whether specific intracellular SPARC interaction partners exist because of the consistent presence of this extracellular matrix protein within muscle cells and regenerating myofibers. To identify these, we performed SPARC pull-down experiments from $\mathrm{C} 2 \mathrm{C} 12$ control and $\mathrm{C} 2 \mathrm{C} 12^{S P A R C} K D$ differentiated cell extracts using beads coupled with SPARC antibody. Knock-down efficiency of SPARC was confirmed at the mRNA (Figure 3A) and protein (Figure 3B) levels.

All proteins were visualized by silver staining, and bands reduced in or absent from $\mathrm{C} 2 \mathrm{C} 12^{S P A R C} K D$ cells were subjected to mass spectrometry for protein identification. As shown in Figure $3 \mathrm{C}$ and identified using mass spectrometry, actin (boxed) is enriched in a SPARC-dependent manner and hence constitutes a potential new SPARC-binding partner.
Actin expression was analyzed in $\mathrm{C} 2 \mathrm{C} 12$ and $\mathrm{C} 2 \mathrm{C} 12^{\text {SPARC KD }}$ cells using a pan-actin antibody, and we observed that SPARC was important for general expression of actin and thus also the structure of the cytoskeleton, which was severely compromised in proliferating $\mathrm{C} 2 \mathrm{C} 12^{S P A R C} K D$ cells (Figure 3D).

Our initial findings of SPARC were based on human tissue; thus, we wanted to investigate whether this interaction was also present in human tissue. We verified the interaction between SPARC and actin in human myoblasts during proliferation and differentiation using the Duolink proximity ligation assay (Figure 4, A and B), suggesting that our previous observation of SPARC in these cells ${ }^{2}$ could be correlated to control of cytoskeletal arrangement during regeneration.

\section{The Interaction between SPARC and Actin Is Directly Correlated with Active Regeneration in Patients with a Muscle Degenerative Condition}

To investigate whether the interaction between SPARC and actin observed in human myoblasts translated into a physiologic setting, we analyzed muscle biopsy specimens from patients with various muscle degenerative disorders, all with a regenerative component. Again using Duolink, we found that the direct interaction between SPARC and actin was present in all types of muscle-damaging disorders analyzed,
A
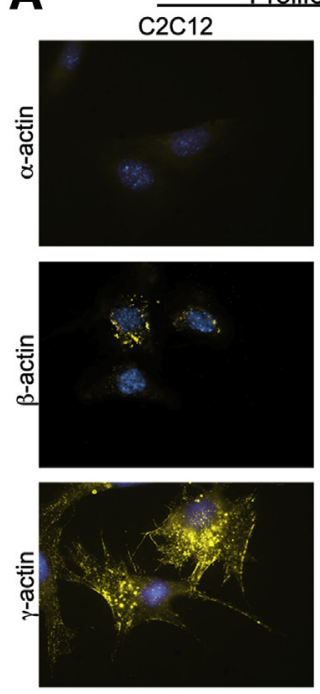

Proliferation
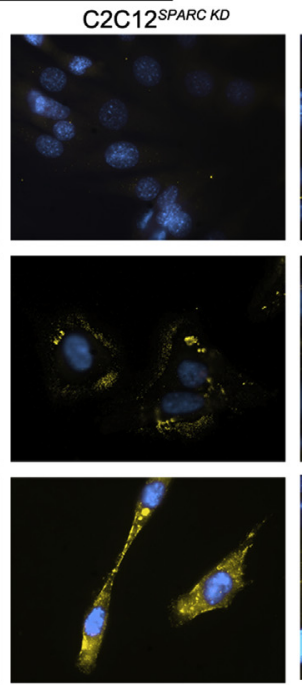
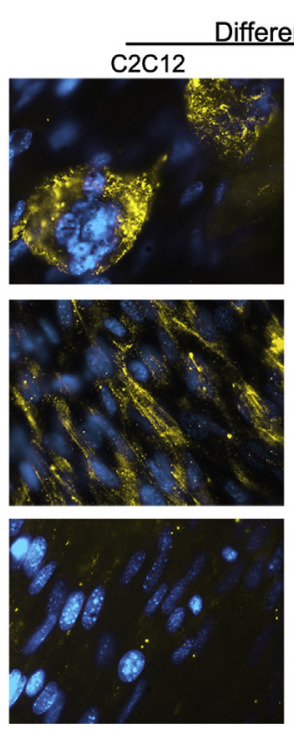

Differentiation
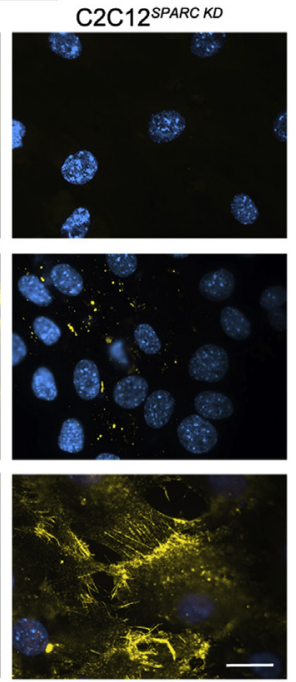

B

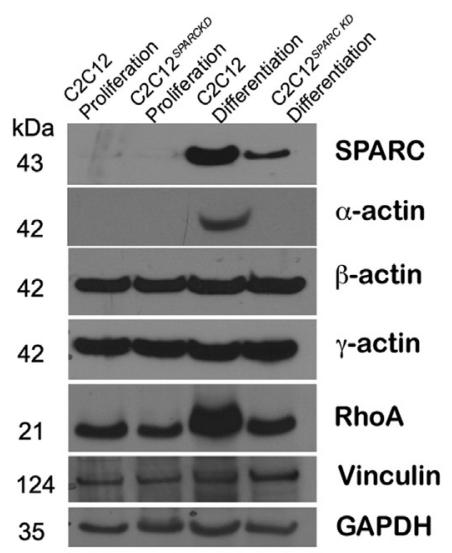

Figure 5 Absence of secreted protein acidic and rich in cysteine (SPARC) affects localization of the three actin isoforms $(\alpha, \beta$, and $\gamma$ ) and the expression of $\alpha$-actin. The observation that SPARC interacts with actin prompted us to investigate in more detail which actin isoforms might be affected by the absence of SPARC. A: $C 2 C 12$ control and C2C12 $2^{\text {SPARC KD }}$ cells were analyzed using immunocytochemistry for the three isoforms $(\alpha, \beta$, and $\gamma)$ during proliferation and differentiation. All three isoforms changed in use observed as changing localization patterns between $C 2 C 12$ control and C2C12 ${ }^{S P A R C} K D$ cells during proliferation and differentiation. B: Western blot was used to investigate expression level of the 3 actin isoforms $(\alpha, \beta$, and $\gamma)$ and expression of SPARC and proteins involved in actin filament formation (RhoA) and focal adhesions (vinculin). Glyceraldehyde-3-phosphate dehydrogenase (GAPDH) was used as loading control. The experiment was run in duplicate and representative images are shown. Western blot confirms the effect of SPARC absence on $\alpha$-actin, which is only expressed in $\mathrm{C}_{2} \mathrm{C} 12$ control cells during differentiation. Expression of both $\beta$ - and $\gamma$-actin appears to remain constant between cell lines and during proliferation and differentiation. Vinculin is not affected by absence of SPARC, whereas RhoA is reduced in C2C12 ${ }^{S P A R C} K D$ cells during differentiation. Scale bar $=20 \mu \mathrm{m}(\mathbf{A})$. 
which included Duchenne muscular dystrophy (Figure 4, C and D), compartment syndrome (Figure 4E), and polymyositis (Figure 4F). Interestingly, the interaction was only present in actively regenerating muscle structures observed using a counterstain for NCAM (Figure 4, C-E). We did not detect the interaction in necrotic areas or in undamaged muscle fibers within the biopsy specimens, suggesting that SPARC function is limited to fibers undergoing remodeling.
A
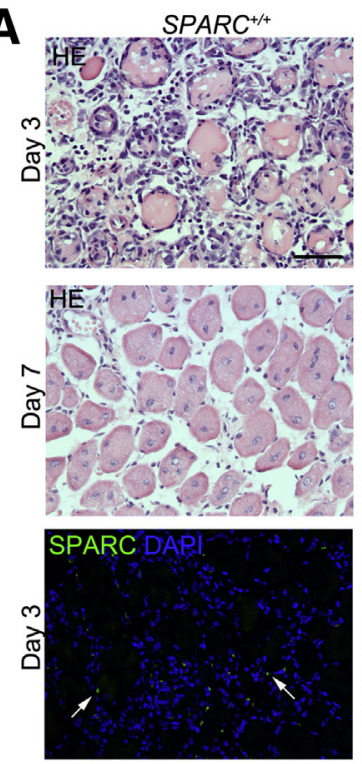

C

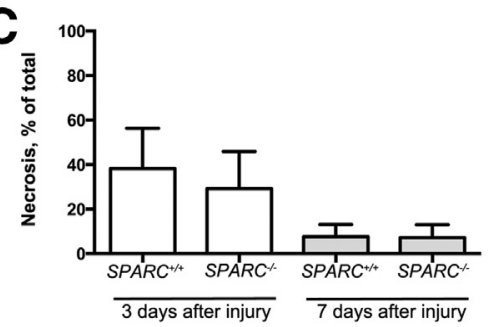

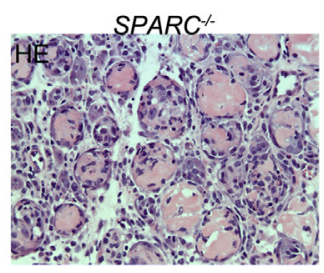
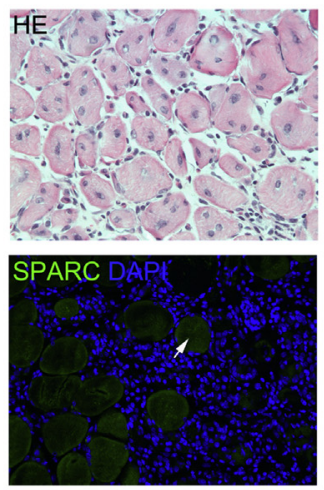

B Cross-sectional area
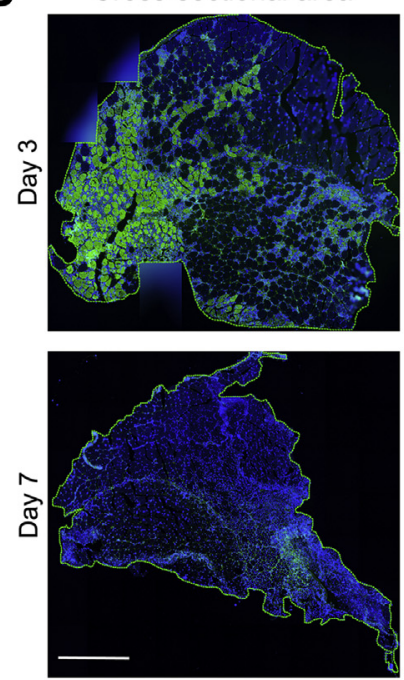

Area of necrosis
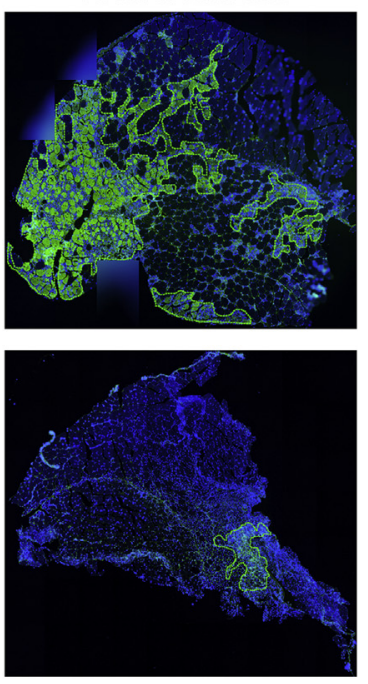

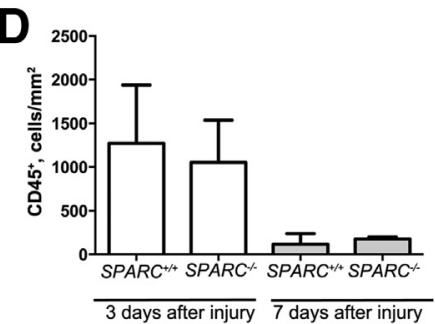

E

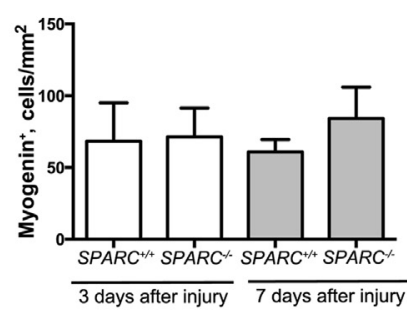

$\mathbf{F}$
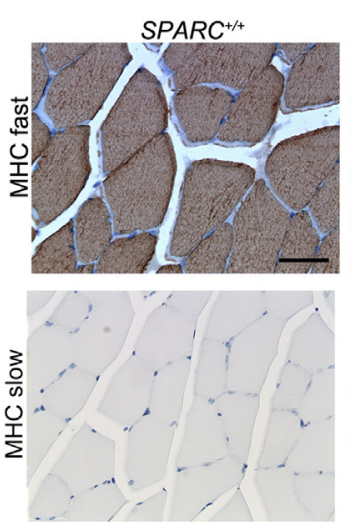

H
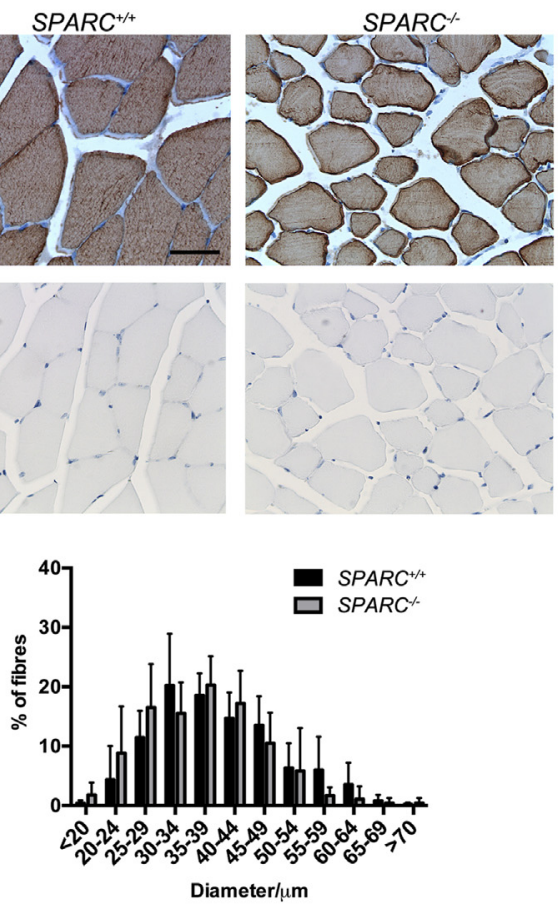

G
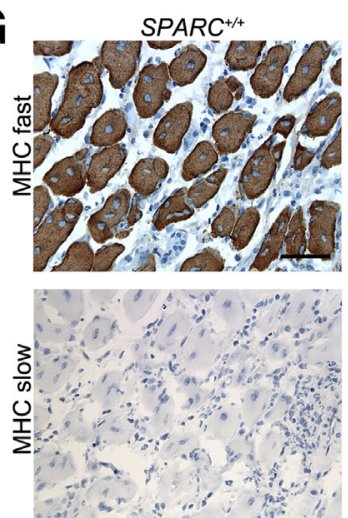

SPARC ${ }^{+/+}$

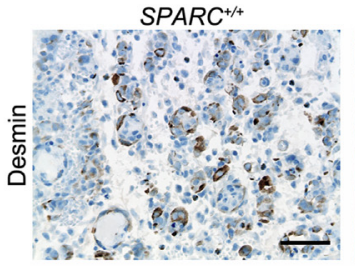

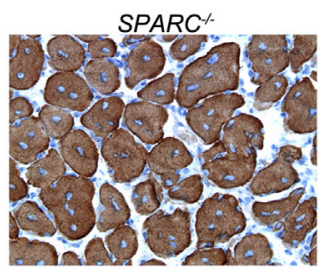
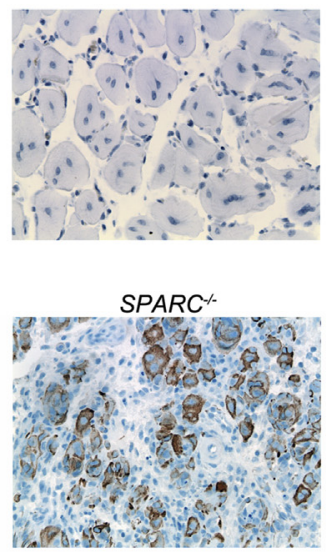
Moreover, control experiments with omission of the primary actin antibody (Figure 4G), the primary SPARC antibody (Figure $4 \mathrm{H}$ ), or both (Figure 4I) confirmed the specificity of the Duolink reaction.

\section{Absence of SPARC Modifies the Expression Pattern of $\alpha-, \beta-$, and $\gamma$-Actin Isoforms during Proliferation and Differentiation of Myoblasts}

Next, we wanted to investigate the expression and localization pattern of the $\alpha-, \beta$-, and $\gamma$-actin isoforms in the presence or absence of SPARC. For this purpose, we used the $\mathrm{C} 2 \mathrm{C} 12$ and $\mathrm{C} 2 \mathrm{C} 12^{\text {SPARC KD }}$ cells. We observed that $\alpha$-actin was only present in very mature differentiating myotubes in the $\mathrm{C} 2 \mathrm{C} 12$ control cells (Figure 5A), and we could not detect $\alpha$-actin in the C2C12 $2^{\text {SPARC KD }}$ cells. This was confirmed by Western blotting of total $\alpha$-actin protein level (Figure 5B). Both cell lines expressed $\beta$-actin (Figure 5, A and B); however, the distribution pattern was different between $\mathrm{C} 2 \mathrm{C} 12$ and $\mathrm{C} 2 \mathrm{C} 12^{S P A R C} K D$. In $\mathrm{C} 2 \mathrm{C} 12$ cells, $\beta$-actin was expressed in a dot-like pattern close to the nuclei during proliferation, and the pattern became more filamentous and elongated and followed the shape of the cells during differentiation. In the $\mathrm{C} 2 \mathrm{C} 12^{S P A R C} K D$ cells, $\beta$-actin was present in a cloud-like pattern located more to the periphery of the cells during proliferation, and during differentiation $\beta$-actin was more diffusely localized. However, as revealed by Western blotting (Figure 5B), $\beta$-actin was expressed equally between the cell lines during both proliferation and differentiation, suggesting that the difference lies in localization. Both cell lines also expressed $\gamma$-actin (Figure 5, A and B), and again there was a marked difference in localization pattern
(Figure 5A) because Western blotting revealed equal amounts of protein present between the cell lines (Figure 5B). In the C2C12 cells, $\gamma$-actin was expressed in the entire cell, clearly marking the cell filaments during proliferation, whereas expression changed markedly during differentiation. In the $\mathrm{C} 2 \mathrm{C} 12^{\text {SPARC KD }}$ cells, $\gamma$-actin was also present in the entire cell during proliferation; however, the expression pattern was not filamentous. This changed during differentiation, where $\gamma$-actin was now expressed in a pattern where cell filaments and contact points were clearly marked. This was in direct contrast to the $\mathrm{C} 2 \mathrm{C} 12$ control cells. The analysis of the different isoforms reveals a clear difference in localization pattern dependent on the presence of SPARC. Moreover, this analysis also reveals that the $\mathrm{C} 2 \mathrm{C} 12$ SPARC KD cells are incapable of forming myotubes during differentiation, and the effect on the isoform expression pattern suggests that in the absence of SPARC, the cells switch to dominantly use the $\gamma$-actin isoform for filament formation because $\alpha$-actin is not expressed at all. In addition to investigating expression levels of the actin isoforms using Western blotting, we further analyzed expression of RhoA, a key regulatory protein for actin stress filament formation, ${ }^{32}$ and vinculin, a focal adhesion protein that interacts with F-actin. ${ }^{33}$ Our results indicate that vinculin expression is not affected by absence of SPARC or regulated between proliferation and differentiation in either $\mathrm{C} 2 \mathrm{C} 12$ or $\mathrm{C} 2 \mathrm{C} 12^{S P A R C} K D$ cells (Figure 5B). However, expression of RhoA is markedly reduced in $\mathrm{C} 2 \mathrm{C} 12^{S P A R C} K D$ during differentiation compared with $\mathrm{C} 2 \mathrm{C} 12$ control cells. Thus, absence of SPARC in vitro appears to affect actin filament formation during differentiation both on the key regulatory protein RhoA and actin isoform use.

\footnotetext{
Figure 6 Skeletal muscle regeneration is not affected by absence of secreted protein acidic and rich in cysteine (SPARC). To investigate whether the absence of SPARC influenced the muscle's ability to regenerate after severe trauma, regeneration was induced in SPARC ${ }^{--}$and SPARC ${ }^{+/+}$mice using a cardiotoxin injection. A: Muscle regeneration was assessed 3 and 7 days after injury using hematoxylin and eosin (H\&E) staining. There was no apparent difference in ability to regenerate based on histologic analysis. The absence of SPARC-positive cells was verified using an immunofluorescence staining against SPARC protein 3 days after injury (bottom left white arrows point toward SPARC-positive cells, and bottom right white arrow points toward unspecific staining of a necrotic myofiber). B: Representative images depicting an entire muscle section from day 3 and day 7 after a cardiotoxin injection. The images show how the entire muscle cross-sectional area and the area of necrosis have been marked for automatic quantification using the VIS software. C: Quantification of the necrotic area following muscle damage. There was no difference in the size of the necrotic area between $S P A R C^{1-}$ and $S P A R C^{+/+}$mice $($day 3 : SPARC $^{-1-}: 29.21 \pm 7.46$ versus SPARC ${ }^{+/+}: 38.21 \pm 9.09, P>0.05$, day $7:$ SPARC $^{-1}: 7.24 \pm 2.89$ versus SPARC $^{+/+}: 7.59 \pm 2.48, P>0.05$, SPARC $^{-/}$ versus $S P A R C^{++}$, unpaired $t$-test). D: The mice were analyzed for ability to elicit an inflammatory response by staining for the common leukocyte marker CD45 followed by quantification. The quantification was performed on the entire section because inflammatory cells are present throughout the muscle tissue due to recruitment and activation from uninjured sites of the tissue as well. There was no difference in presence of inflammatory cells between SPARC ${ }^{-1}$

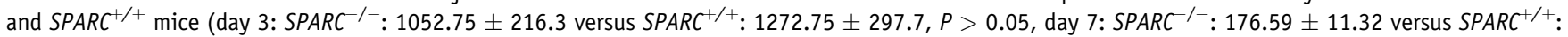
$114.60 \pm 55.57, P>0.05$, SPARC $^{-1-}$ versus SPARC ${ }^{+/+}$, unpaired $t$-test). E: The mice were analyzed for the presence of myogenin-positive nuclei following regeneration to assess the myoblasts' ability to enter differentiation during regeneration. This quantification was performed in the regenerative area only to ascertain that any differences in size of the lesions would not affect the result. There was no difference in the number of myogenin-positive nuclei in the regenerative area between SPARC $^{-/-}$and PPARC $^{+/+}$mice (day 3: SPARC $^{-1-}: 71.33 \pm 8.99$ versus SPARC ${ }^{+/+}: 68.29 \pm 11.99, P>0.05$, day $7:$ SPARC $^{/-}$: $84.23 \pm 9.71$ versus $\operatorname{SPARC}^{+/+}: 60.83 \pm 3.83, P>0.05$, SPARC $^{-/-}$versus $S P A R C^{+/+}$, unpaired $t$-test). F: The contralateral, uninjured tibialis anterior (TA) muscles were analyzed for fiber phenotypes, and it was observed that both $S P A R C^{-/}$and $S P A R C^{+/+}$mice predominantly expressed type 2 myosin heavy chain (MHC) fast-positive fibers. G: The TA muscles 7 days after cardiotoxin injection was further examined for fiber phenotypes to investigate whether any fiber type switching had occurred during regeneration in $\mathrm{SPARC}^{-1-}$ versus $S P A R \mathrm{C}^{+/+}$mice. No fiber-type switching was observed, and all regenerative fibers expressed MHC fast. H: The contralateral, uninjured control TA muscles were further analyzed to determine fiber diameters. There was no difference in myofiber size between $\operatorname{SPARC}^{-1-}$ and SPARC ${ }^{+/+}$mice $(P>0.05,2$-way analysis of variance). I: In support of the morphologic and morphometrical analyses of regeneration, desmin staining was performed to visualize the presence of actively regenerating myoblasts and the formation of new myofibers in a similar manner between SPARC $^{-/-}$and SPARC ${ }^{+/+}$mice 3 days after cardiotoxin injection. Data are expressed as means \pm SEM $(\mathbf{C}-\mathbf{E}, \mathbf{H})$. Scale bars $=50 \mu \mathrm{m}(\mathbf{A}, \mathbf{F}, \mathbf{G}, \mathbf{I}) ; 500 \mu \mathrm{m}(\mathbf{B})$.
} 

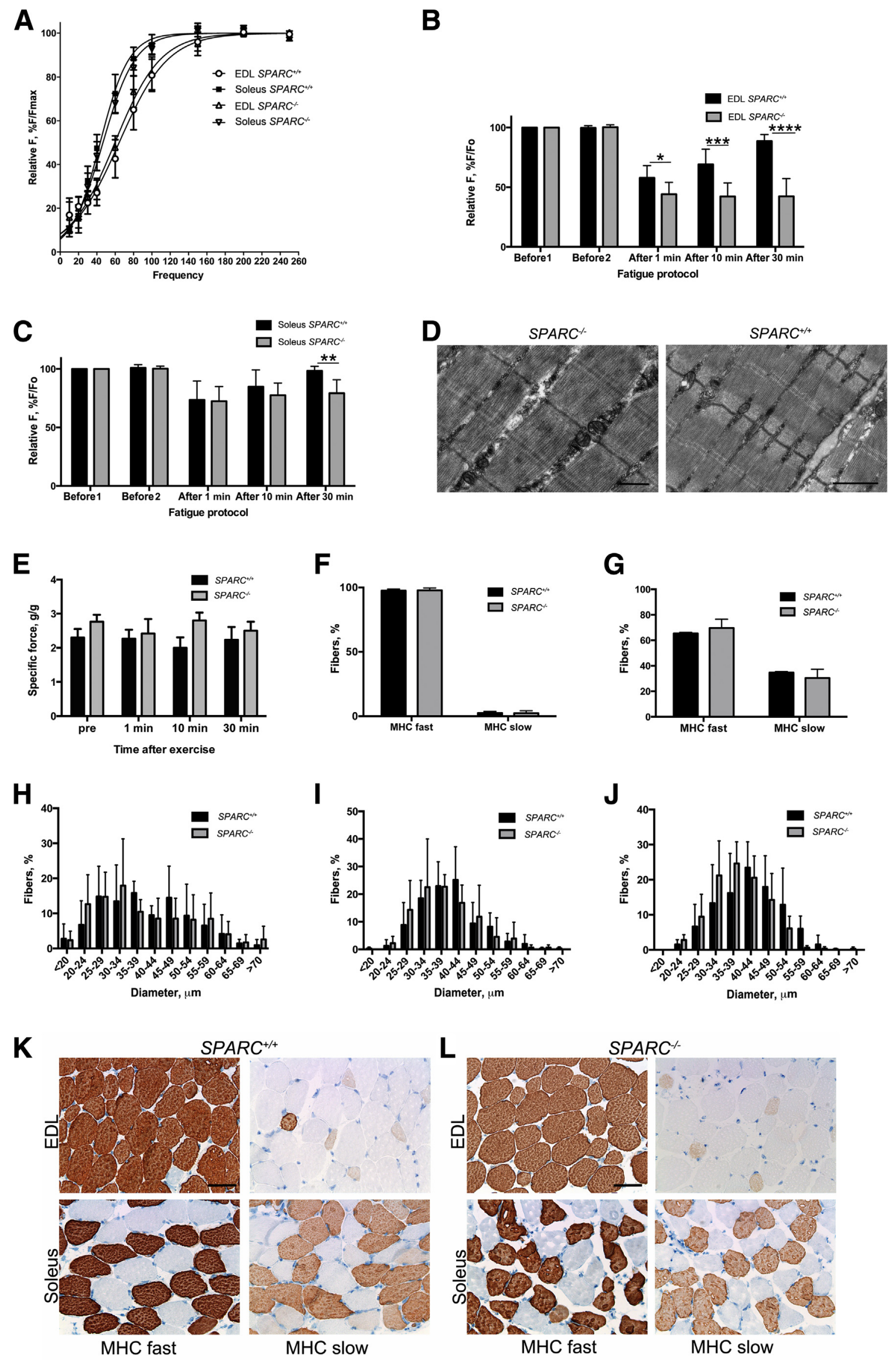


\section{$\mathrm{SPARC}^{-/-}$Mice Are Fully Capable of Undergoing Regeneration in Vivo}

The effect on isoform expression in vitro prompted us to investigate whether lack of SPARC directly inhibits skeletal muscle regeneration in vivo. For this purpose, we used $S P A R C^{-/-}$mice and SPARC ${ }^{+/+}$littermate controls. These mice had a cardiotoxin injection in the right TA muscle and were analyzed for the ability to regenerate after 3 and 7 days. We observed no histologic difference (hematoxylin and eosin staining) in regenerative ability between $S P A R C^{-1-}$ and SPARC ${ }^{+/+}$mice (Figure 6A). We analyzed the ability to regenerate further by investigating the amount of necrosis (Figure 6, B and C), the elicited inflammatory response (Figure 6D), and the number of myogenin-positive nuclei (Figure 6D) as a marker for actively differentiating myoblasts. We found that none of these parameters were affected by the absence of SPARC. We also performed a desmin staining to further evaluate the regenerative ability (Figure 6I), and we observed that already 3 days after injury there was presence of desmin-positive cells in both $S P A R C^{-/-}$and SPARC $C^{+/+}$mice, supporting that absence of SPARC does not affect regeneration.

Fiber-type analysis revealed that there was no difference in fiber-type composition in uninjured, contralateral muscles between $S P A R C^{+/+}$and SPARC ${ }^{-/-}$mice. TA muscle from both presented predominantly with MHC fast fibers (Figure 6F), and there was no difference in fiber diameter (Figure 6H). Moreover, fiber-type analysis of the regenerating muscles revealed that after injury and during regeneration no isotype shift occurred; thus, the regenerating fibers also predominantly expressed MHC fast (Figure 6G).

The SPARC $C^{-1-}$ phenotype was verified (in addition to genotyping PCR, not shown) by the absence of SPARC-positive cells in the regenerating area (day 3) in $S P A R C^{-1-}$ mice compared with SPARC ${ }^{+/+}$mice (Figure 6A). This finding suggested that even though muscle cells lacking SPARC in vitro had a disturbed actin cytoskeleton, this did not translate to an in vivo setting, indicating that there might be compensatory mechanisms present in vivo.

\section{Lack of SPARC in Skeletal Muscle Causes Inability to Recover following Fatigue in Vitro}

To further investigate the physiologic role of SPARC within skeletal muscle, we analyzed muscle function using in vitro force measurements on EDL and soleus muscles from $S P A R C^{-/-}$and SPARC ${ }^{+/+}$mice. There were no differences in force-frequency relationship between $S P A R C^{-/-}$and $S P A R C^{+/+}$mice for either EDL $\left(\mathrm{F}_{50}=65 \mathrm{~Hz}\right.$ for $S P A R C^{+/+}, \mathrm{F}_{50}=59 \mathrm{~Hz}$ for $S P A R C^{-/-}$) or soleus $\left(\mathrm{F}_{50}=43 \mathrm{~Hz}\right.$ for $S P A R C^{+/+}, \mathrm{F}_{50}=46 \mathrm{~Hz}$ for $\left.S P A R C^{-/-}\right)$ (Figure 7A). Furthermore, there was no difference in the maximum tetanic force between $S P A R C^{-/-}$and $S P A R C^{+/+}$ mice for either EDL or soleus. Thus, the force-generating ability in resting, uninjured muscles appeared to be independent of SPARC, which also correlates with the observation that SPARC is not detectable in normal, healthy, uninjured muscle. ${ }^{2}$ However, after the 3-minute fatigue stimulation protocol, SPARC ${ }^{-1-}$ mice had a significant impairment of force recovery for both EDL (Figure 7B) and soleus (Figure $7 \mathrm{C}$ ), with $S P A R C^{+/+}$mice fully recovering within 30 minutes. To investigate this force recovery defect, we analyzed EDL and soleus muscles from SPARC ${ }^{-1-}$ and $S P A R C^{+/+}$mice using electron microscopy (Figure 7D). We did not observe any difference in sarcomere structure and found no abnormalities in SPARC ${ }^{-1-}$ mice in undamaged muscle, which correlated well with the normal ability to generate force. Next, we wanted to investigate whether this force deficit after fatigue translated into an in vivo setting. We therefore subjected $S P A R C^{+/+}$and $S P A R C^{-/-}$mice to eccentric exercise on a treadmill and measured force grip strength before exercise and at 1, 10, and 30 minutes after exercise. We observed that the short-term exercise protocol did not fatigue the mice in a manner that caused any decrease in the ability to elicit force, and there was no difference between $S P A R C^{+/+}$and $S P A R C^{-1-}$ mice in their ability to generate force in vivo either before or after short-term exercise (Figure 7E). To further analyze the background for the in vitro force decrease results, we investigated the muscle phenotypes in $S P A R C^{+/+}$and $S P A R C^{-/-}$using $\mathrm{MHC}$ fast and slow staining and

\footnotetext{
Figure 7 Recovery of force in vitro is compromised in secreted protein acidic and rich in cysteine (SPARC) knockout mice (SPARC ${ }^{-1}$ ) mice after fatigue. To test whether absence of SPARC had an influence on force transmission in skeletal muscle, we analyzed extensor digitorum longus (EDL) and soleus muscle using in vitro force measurements. A: Force-frequency analysis of EDL and soleus muscles revealed no difference between SPARC $C^{--}$and SPARC $C^{+/+}$mice. The ability to generate force after a fatigue protocol was analyzed in EDL (B) and soleus (C) muscles from SPARC ${ }^{/-}$and SPARC ${ }^{+/+}$mice. There was a significant difference in the ability to recover force generation in $S P A C^{-1-}$ in EDL and soleus. D: Electron microscopy of representative EDL muscle from SPARC ${ }^{/-}$and $S P A R C^{+/+}$mice showing normal sarcomeric units in both. E: On the basis of the results in vitro, we tested if a short-term eccentric exercise protocol-induced fatigue earlier in $S P A R C^{-1-}$ mice and whether there was a similar decrease in force grip of the front paws combined with inability to recover. We observed that the short-term exercise protocol did not induce measurable fatigue and therefore also no deficit in recovery between SPARC ${ }^{/-}$and SPARC ${ }^{+/+}$mice. To further investigate the EDL and soleus muscle phenotypes, we performed analyses of fiber types in EDL (F) and soleus (G) muscles. We observed no difference in fiber types between SPARC $^{-1-}$ and SPARC ${ }^{+/+}$mice in $\operatorname{EDL}(P>0.05,2$-way analysis of variance, Sidak's multiple comparison) or soleus $(P>0.05,2-$ way analysis of variance, Sidak's multiple comparison). We further analyzed the muscles by measuring diameters of all EDL fibers (H), myosin heavy chain (MHC) fast-positive fibers in soleus (I), and MHC slow-positive fibers in soleus $(\mathbf{J})$. We did not detect any differences in fiber diameters between SPARC ${ }^{-1}$ and SPARC $^{+/+}$mice $(P>0.05,2$-way analysis of variance for all analyses) $(\mathbf{H}-\mathbf{J})$. In support of the quantifications in $\mathbf{F}$ and $\mathbf{G}$, we show representative images of the MHC fast and MHC slow staining of EDL and soleus muscles in $\operatorname{SPARC}^{+/+}$mice $(\mathbf{K})$ and $S P A R C^{-/-}$mice $(\mathbf{L}) .{ }^{*} P<0.05$ at 1 minute versus $S P A R C^{+/+} ;{ }^{* *} P<0.01$ at 30 minutes versus SPARC $C^{+/+}$(multiple $t$-tests); ${ }^{* * *} P<0.001$ at 10 minutes versus SPARC ${ }^{+/+} ;{ }^{* * * * P}<0.0001$ at 30 minutes versus $S P A R C^{+/+}$(multiple $t$-tests). Scale bars $=500 \mathrm{~nm}$ (left panel); $1000 \mathrm{~nm}$ (right panel) (D); $50 \mu \mathrm{m}$ (K and L).
} 
measurements of fiber diameters of both EDL and soleus muscle. There was no difference in fiber-type composition (Figure 7, F, G, K, and L) or diameter (Figure 7, H-J) of either EDL or soleus between SPARC $C^{+/+}$and SPARC ${ }^{-/-}$; thus, the decrease in force after the in vitro protocol is not based on differences in fiber types or diameters and must relate to other intrinsic effects dependent on SPARC within the muscle tissue, which most likely are compensated in vivo.

\section{Discussion}

SPARC is an evolutionarily conserved protein among all phyla $^{34}$ and belongs to a group of extracellular matrix proteins that are regulatory but do not contribute significantly to extracellular matrix structure. ${ }^{35}$ The fact that SPARC is evolutionarily conserved and expressed by multiple species suggests that this protein has important regulatory role(s). Our previous finding of SPARC expression during muscle development and in patients with myopathy ${ }^{2}$ is consistent with the expression pattern of matricellular proteins, which are generally present during embryogenesis, not expressed in adult tissues but reexpressed in damaged or remodeling tissues, as well as in response to inflammation or cancers. ${ }^{35} \mathrm{In}$ line with this, SPARC is expressed in a regulated manner during regeneration in mice as well. ${ }^{20}$ In more primitive species SPARC function in skeletal muscle has been associated with reduced motility and developmental muscle defects, ${ }^{18,19}$ whereas in mice, absence of SPARC has previously not been correlated with a muscle phenotype. ${ }^{14}$

However, the exact role of SPARC within mammalian skeletal muscle tissue remains unknown because the presence of SPARC in single cells and in developing or regenerating myofibers does not correlate with previously described general effects of SPARC, such as inhibition of proliferation ${ }^{36}$ and collagen deposition in connective tissue. ${ }^{37}$

In the current study, we therefore investigated the role of SPARC in skeletal muscle tissue. We made several interesting observations: i) SPARC interacts directly with actin in myoblasts, and lack of SPARC results in a changed localization pattern of the different actin isoforms in vitro; ii) SPARC interacts directly with actin in the regenerating myofibers in patients with muscular dystrophies and myopathies; iii) SPARC absence in mice results in defects in force recovery after a fatigue stimulation protocol in vitro; and iv) SPARC is not necessary for the regeneration process after muscle damage with toxin that completely destroys the myofibers. Because SPARC is not expressed by normal, adult healthy muscle, ${ }^{2}$ our results indicate that the function of SPARC in skeletal muscle is temporary, occurring only during remodeling or regeneration of the skeletal muscle with a possible physiologic role to recover the ability to generate force after damage. The indication that the function of SPARC is temporary suggests that SPARC needs to be synthesized according to need and maybe even be transported from the extracellular to the intracellular compartment. This agrees with the observed shuttling mechanism of SPARC in fibroblasts. ${ }^{22}$

SPARC protein structure has been extensively studied, and it is well known that SPARC contains an extracellular $\mathrm{Ca}^{2+}$ domain containing $2 \mathrm{EF}-$ hand motifs, ${ }^{38}$ a follistatinlike domain and a kazal-like domain. ${ }^{39}$ We have analyzed this protein for presence of conserved actin-binding domain(s) using the Conserved Domain Database, National Center for Biotechnology Information, ${ }^{40}$ and the only other conserved domains were a collagen binding site and a putative $\mathrm{K}^{+}$-binding site. Thus, SPARC is not a classic actinbinding protein, suggesting that a more general mechanism is involved in the specific interaction between actin and SPARC. The role of SPARC as a molecular chaperone has been suggested previously, where it is proposed that SPARC is a scavenger chaperone mediating disassembly of the extracellular matrix networks. ${ }^{22}$ SPARC has chaperonelike activity in a thermal aggregation assay. Here, SPARC remained stable at $50^{\circ} \mathrm{C}$, where it was capable of protecting alcohol dehydrogenase from temperature-induced aggregation. ${ }^{26}$ In line with this, our results could suggest a protective chaperone-like activity of SPARC in remodeling skeletal muscle.

Interestingly, the interaction between SPARC and actin is present in such diverse muscular disorders as Duchenne muscular dystrophy, a genetic disorder with mutated dystrophin, ${ }^{41}$ polymyositis, an autoimmune inflammatory disorder that affects the skeletal muscles ${ }^{42}$ and compartment syndrome, an illness occurring in response to compromised blood supply to the muscle. ${ }^{43}$ This finding suggests that the interaction between these proteins represents a common mechanism related to muscle damage and not a specific type of injury.

During muscle cell differentiation, major changes in cytoskeletal architecture occur, involving all actin isoforms, and during formation of myotubes there is a shift in expression and localization pattern from $\beta$ - and $\gamma$-actin to $\alpha$ actin. ${ }^{44,45}$ In agreement with this, we observed in the $\mathrm{C} 2 \mathrm{C} 12$ control cells that $\beta$ - and $\gamma$-actins are predominantly expressed during proliferation but with very different localization patterns. Where $\gamma$-actin clearly outlines the cytoskeletal structure, $\beta$-actin is present in a more dot-like pattern. During myotube formation, $\alpha$-actin is now highly expressed in mature myotubes, whereas $\beta$ - and $\gamma$-actin are still present but change the localization pattern during differentiation. These morphologic actin changes are not observed in the $\mathrm{C} 2 \mathrm{C} 12^{S P A R C ~ K D}$ cells. $\beta$-actin is present in a cloud-like pattern and is barely observable during differentiation, and $\alpha$-actin is not detectable at all. The fact that we do not see $\alpha$-actin could be a result of the $\mathrm{C} 2 \mathrm{C} 12^{\text {SPARC }}$ $K D$ cells inability to differentiate and form myotubes. However, it might be the lack of interaction between SPARC and actin that results in the inability of the $\mathrm{C} 2 \mathrm{C} 12^{S P A R C} K D$ cells to differentiate and hence the absence of $\alpha$-actin expression in $\mathrm{C} 2 \mathrm{C} 12^{S P A R C} K D$ cells. Interestingly, the $\mathrm{C} 2 \mathrm{C} 12^{S P A R C} K D$ cells appear to compensate for the lack 
of SPARC by expressing and using $\gamma$-actin during differentiation. In the proliferative state, $\gamma$-actin is present throughout the cells, but the localization pattern suggests that these cells have a compromised cytoskeletal structure. However, during differentiation, this changes, and $\gamma$-actin reveals a cytoskeletal network both inside cells and between cells, suggesting that during differentiation these cells compensate through the use of $\gamma$-actin to form filaments and attach to each other instead of $\alpha$-actin. Interestingly, though, the amount of $\gamma$-actin remains the same during proliferation and differentiation, suggesting that it is most likely use and localization that are changed. The fact that $\mathrm{C} 2 \mathrm{C} 12^{S P A R C K D}$ uses $\gamma$-actin in this manner is exciting because $\gamma$-actin is generally considered one of the nonmuscle actins, yet $\gamma$-actin is a component of the $\mathrm{z}$ disk. ${ }^{46}$

Furthermore, we detect the direct interaction between actin and SPARC during both proliferation and differentiation of primary derived human muscle cells. This observation, together with the shift in isoform localization and use in response to lack of SPARC, points toward SPARC as a protector or stabilizer of the cytoskeleton during myogenesis.

The obvious effect of lack of SPARC on differentiation and localization of the actin isoforms is interesting, considering that we previously reported an inhibition of differentiation in $\mathrm{C} 2 \mathrm{C} 12$ cells when SPARC was overexpressed in these cells. ${ }^{20}$ This finding suggests that expression of SPARC during differentiation needs to be well regulated possibly to aid actin during myogenesis. It has recently been found that SPARC has a specific effect on collagen deposition in the basement membrane. Under normal circumstances, SPARC appears to facilitate collagen trafficking from sites of production to sites not expressing collagen; however, when SPARC is overexpressed, the effect is disrupted, and collagen deposition into the basement membrane is reduced, thus facilitating invasive processes instead. $^{47}$ Therefore, regardless of cellular context, it appears that SPARC needs to be tightly controlled to avoid detrimental effects.

On the basis of the inability of $\mathrm{C} 2 \mathrm{C} 12^{S P A R C} K D$ cells to differentiate, SPARC ${ }^{-1-}$ mice would be expected to have a regenerative defect; however, this is not the case. We also did not observe any cytoskeletal defects with electron microscopy in skeletal muscle from SPARC ${ }^{-1-}$ mice, which have normal sarcomeric units. These mice are also capable of generating normal force; thus, the effect of SPARC on force appears not to be related to normal function and general force generation. This is further implied by our results from the short-term, eccentric exercise study, which indicates that $S P A R C^{-1-}$ do not fatigue faster in vivo; thus, redundant systems could be in play.

Because our short-term exercise protocol was not capable of inducing fatigue that was measurable using force grip analysis in either SPARC ${ }^{+/+}$or SPARC ${ }^{-/-}$, this issue needs to be addressed further, possibly through long-term exercise studies combined with strength analyses over time. On the basis of the in vivo results from the regeneration study and the exercise and force grip study, it appears that the isolated effects of SPARC observed in vitro, such as the changes in the cytoskeleton, inhibition of differentiation, and force decrease after fatigue, are not transferable to an in vivo setting, pinpointing the fact that compensatory mechanisms must be present in vivo.

In summary, this study provides new insight into the mechanistic function of SPARC in skeletal muscle and highlights how a multidomain protein can exert very different functions, depending on the compartmental presence and the tissue or disease context in which it is expressed.

\section{Acknowledgments}

We thank Karin Trampedach and Kirsten Hansen, Department of Pathology, Odense University Hospital, for technical assistance with electron microscopy.

L.H.J. planned all and performed most experiments and wrote the manuscript; P.L.J. performed the regeneration study and morphologic studies on fiber types and diameters; A.B. and J.M.-J. assisted with the mass spectrometry experiments and analyzed the PMFs and MALDI-TOF/TOF data; L.B.D. and D.R. stained and counted sections from $m d x$ and wt mice and performed the analysis of the data; J.S. isolated the human myoblasts; L.G.H. and N.Ø. performed the in vitro force measurements and analyzed the data; L.B.D. and L.H.J. performed the exercise study and force grip measurements and analyzed the data; H.L. supervised the generation of SPARC clones; and H.D.S. provided patient samples and assisted in analysis of the data. All contributing authors assisted in writing and correcting the manuscript.

\section{References}

1. Flanigan KM: The muscular dystrophies. Semin Neurol 2012, 32: 255-263

2. Jorgensen LH, Petersson SJ, Sellathurai J, Andersen DC, Thayssen S, Sant DJ, Jensen CH, Schroder HD: Secreted protein acidic and rich in cysteine (SPARC) in human skeletal muscle. J Histochem Cytochem 2009, 57:29-39

3. Brekken RA, Sage EH: SPARC, a matricellular protein: at the crossroads of cell-matrix communication. Matrix Biol 2001, 19:816-827

4. Jejurikar SS, Kuzon WM Jr: Satellite cell depletion in degenerative skeletal muscle. Apoptosis 2003, 8:573-578

5. Zhou X, Tan FK, Guo X, Arnett FC: Attenuation of collagen production with small interfering RNA of SPARC in cultured fibroblasts from the skin of patients with scleroderma. Arthritis Rheum 2006, 54:2626-2631

6. Demopoulos K, Arvanitis DA, Vassilakis DA, Siafakas NM, Spandidos DA: MYCL1, FHIT, SPARC, p16(INK4) and TP53 genes associated to lung cancer in idiopathic pulmonary fibrosis. J Cell Mol Med 2002, 6:215-222

7. Atorrasagasti C, Peixoto E, Aquino JB, Kippes N, Malvicini M, Alaniz L, Garcia M, Piccioni F, Fiore EJ, Bayo J, Bataller R, Guruceaga E, Corrales F, Podhajcer O, Mazzolini G: Lack of the matricellular protein SPARC (secreted protein, acidic and rich in cysteine) attenuates liver fibrogenesis in mice. PLoS One 2013, 8:e54962

8. Kos K, Wilding JP: SPARC: a key player in the pathologies associated with obesity and diabetes. Nat Rev Endocrinol 2010, 6:225-235 
9. Blazejewski S, Le BB, Boussarie L, Blanc JF, Malaval L, Okubo K, Saric J, Bioulac-Sage P, Rosenbaum J: Osteonectin (SPARC) expression in human liver and in cultured human liver myofibroblasts. Am J Pathol 1997, 151:651-657

10. McCurdy S, Baicu CF, Heymans S, Bradshaw AD: Cardiac extracellular matrix remodeling: fibrillar collagens and Secreted Protein Acidic and Rich in Cysteine (SPARC). J Mol Cell Cardiol 2010, 48:544-549

11. Mayer U, Aumailley M, Mann K, Timpl R, Engel J: Calcium-dependent binding of basement membrane protein BM-40 (osteonectin, SPARC) to basement membrane collagen type IV. Eur J Biochem 1991, 198:141-150

12. Sage EH, Bornstein P: Extracellular proteins that modulate cell-matrix interactions. SPARC, tenascin, and thrombospondin. J Biol Chem 1991, 266:14831-14834

13. Sage H, Decker J, Funk S, Chow M: SPARC: a Ca2+-binding extracellular protein associated with endothelial cell injury and proliferation. J Mol Cell Cardiol 1989, 21 Suppl 1:13-22

14. Gilmour DT, Lyon GJ, Carlton MB, Sanes JR, Cunningham JM, Anderson JR, Hogan BL, Evans MJ, Colledge WH: Mice deficient for the secreted glycoprotein SPARC/osteonectin/BM40 develop normally but show severe age-onset cataract formation and disruption of the lens. EMBO J 1998, 17:1860-1870

15. Delany AM, Amling M, Priemel M, Howe C, Baron R, Canalis E: Osteopenia and decreased bone formation in osteonectin-deficient mice. J Clin Invest 2000, 105:1325

16. Bradshaw AD, Graves DC, Motamed K, Sage EH: SPARC-null mice exhibit increased adiposity without significant differences in overall body weight. Proc Natl Acad Sci U S A 2003, 100:6045-6050

17. Nakamura K, Yamanouchi K, Nishihara M: Secreted protein acidic and rich in cysteine internalization and its age-related alterations in skeletal muscle progenitor cells. Aging Cell 2014, 13:175-184

18. Purcell L, Gruia-Gray J, Scanga S, Ringuette M: Developmental anomalies of Xenopus embryos following microinjection of SPARC antibodies. J Exp Zool 1993, 265:153-164

19. Schwarzbauer JE, Spencer CS: The Caenorhabditis elegans homologue of the extracellular calcium binding protein SPARC/osteonectin affects nematode body morphology and mobility. Mol Biol Cell 1993, 4:941-952

20. Petersson SJ, Jorgensen LH, Andersen DC, Norgaard RC, Jensen CH, Schroder HD: SPARC is up-regulated during skeletal muscle regeneration and inhibits myoblast differentiation. Histol Histopathol 2013, 28:1451-1460

21. Motamed K, Blake DJ, Angello JC, Allen BL, Rapraeger AC, Hauschka SD, Sage EH: Fibroblast growth factor receptor-1 mediates the inhibition of endothelial cell proliferation and the promotion of skeletal myoblast differentiation by SPARC: a role for protein kinase A. J Cell Biochem 2003, 90:408-423

22. Chlenski A, Guerrero LJ, Salwen HR, Yang Q, Tian Y, Morales La Madrid A, Mirzoeva S, Bouyer PG, Xu D, Walker M, Cohn SL: Secreted protein acidic and rich in cysteine is a matrix scavenger chaperone. PLoS One 2011, 6:e23880

23. Weaver MS, Workman G, Sage EH: The copper binding domain of SPARC mediates cell survival in vitro via interaction with integrin beta1 and activation of integrin-linked kinase. J Biol Chem 2008, 283: 22826-22837

24. Fenouille N, Puissant A, Dufies M, Robert G, Jacquel A, Ohanna M, Deckert M, Pasquet JM, Mahon FX, Cassuto JP, Raynaud S, TartareDeckert S, Auberger P: Persistent activation of the Fyn/ERK kinase signaling axis mediates imatinib resistance in chronic myelogenous leukemia cells through upregulation of intracellular SPARC. Cancer Res 2010, 70:9659-9670

25. Fenouille N, Puissant A, Tichet M, Zimniak G, Abbe P, Mallavialle A, Rocchi S, Ortonne JP, Deckert M, Ballotti R, Tartare-Deckert S: SPARC functions as an anti-stress factor by inactivating p53 through Akt-mediated MDM2 phosphorylation to promote melanoma cell survival. Oncogene 2011, 30:4887-4900

26. Emerson RO, Sage EH, Ghosh JG, Clark JI: Chaperone-like activity revealed in the matricellular protein SPARC. J Cell Biochem 2006, 98 : 701-705
27. Kudo H, Hirayoshi K, Kitagawa Y, Imamura S, Nagata K: Two collagen-binding proteins, osteonectin and HSP47, are coordinately induced in transformed keratinocytes by heat and other stresses. Exp Cell Res 1994, 212:219-224

28. Neri M, Descalzi-Cancedda F, Cancedda R: Heat-shock response in cultured chick embryo chondrocytes. Osteonectin is a secreted heatshock protein. Eur J Biochem 1992, 205:569-574

29. Gaster M, Beck-Nielsen H, Schroder HD: Proliferation conditions for human satellite cells: the fractional content of satellite cells. APMIS 2001, 109:726-734

30. Vandesompele J, De Preter K, Pattyn F, Poppe B, Van Roy N, De Paepe A, Speleman F: Accurate normalization of real-time quantitative RT-PCR data by geometric averaging of multiple internal control genes. Genome Biol 2002, 3. RESEARCH0034

31. Lefaucheur JP, Pastoret C, Sebille A: Phenotype of dystrophinopathy in old mdx mice. Anat Rec 1995, 242:70-76

32. Maekawa M, Ishizaki T, Boku S, Watanabe N, Fujita A, Iwamatsu A, Obinata T, Ohashi K, Mizuno K, Narumiya S: Signaling from Rho to the actin cytoskeleton through protein kinases ROCK and LIM-kinase. Science 1999, 285:895-898

33. Thompson PM, Tolbert CE, Campbell SL: Vinculin and metavinculin: oligomerization and interactions with F-actin. FEBS Lett 2013, 587: $1220-1229$

34. Martinek N, Zou R, Berg M, Sodek J, Ringuette M: Evolutionary conservation and association of SPARC with the basal lamina in Drosophila. Dev Genes Evol 2002, 212:124-133

35. Bornstein P, Sage EH: Matricellular proteins: extracellular modulators of cell function. Curr Opin Cell Biol 2002, 14:608-616

36. Sage EH, Bassuk JA, Yost JC, Folkman MJ, Lane TF: Inhibition of endothelial cell proliferation by SPARC is mediated through a $\mathrm{Ca}(2+)-$ binding EF-hand sequence. J Cell Biochem 1995, 57:127-140

37. Rentz TJ, Poobalarahi F, Bornstein P, Sage EH, Bradshaw AD: SPARC regulates processing of procollagen I and collagen fibrillogenesis in dermal fibroblasts. J Biol Chem 2007, 282: 22062-22071

38. Hohenester E, Maurer P, Hohenadl C, Timpl R, Jansonius JN, Engel J: Structure of a novel extracellular $\mathrm{Ca}(2+)$-binding module in $\mathrm{BM}-40$. Nat Struct Biol 1996, 3:67-73

39. Hohenester E, Maurer P, Timpl R: Crystal structure of a pair of follistatin-like and EF-hand calcium-binding domains in BM-40. EMBO J 1997, 16:3778-3786

40. Marchler-Bauer A, Zheng C, Chitsaz F, Derbyshire MK, Geer LY, Geer RC, Gonzales NR, Gwadz M, Hurwitz DI, Lanczycki CJ, Lu F, Lu S, Marchler GH, Song JS, Thanki N, Yamashita RA, Zhang D, Bryant SH: CDD: conserved domains and protein three-dimensional structure. Nucleic Acids Res 2013, 41:D348-D352

41. Hoffman EP, Brown RH Jr, Kunkel LM: Dystrophin: the protein product of the Duchenne muscular dystrophy locus. Cell 1987, 51:919-928

42. Dimachkie MM, Barohn RJ, Amato AA: Idiopathic Inflammatory Myopathies. Neurol Clin 2014, 32:595-628

43. Qvarfordt P, Christenson JT, Eklof B, Ohlin P, Saltin B: Intramuscular pressure, muscle blood flow, and skeletal muscle metabolism in chronic anterior tibial compartment syndrome. Clin Orthop Relat Res 1983:284-290

44. Gunning P, Hardeman E, Wade R, Ponte P, Bains W, Blau HM, Kedes L: Differential patterns of transcript accumulation during human myogenesis. Mol Cell Biol 1987, 7:4100-4114

45. Hayward LJ, Schwartz RJ: Sequential expression of chicken actin genes during myogenesis. J Cell Biol 1986, 102:1485-1493

46. Papponen H, Kaisto T, Leinonen S, Kaakinen M, Metsikko K: Evidence for gamma-actin as a $\mathrm{Z}$ disc component in skeletal myofibers. Exp Cell Res 2009, 315:218-225

47. Morrissey MA, Jayadev R, Miley GR, Blebea CA, Chi Q, Ihara S, Sherwood DR: SPARC Promotes Cell Invasion In Vivo by Decreasing Type IV Collagen Levels in the Basement Membrane. PLoS Genet 2016, 12:e1005905 